\title{
Computation of scattering from $N$ spheres using multipole reexpansion
}

\author{
Nail A. Gumerov* and Ramani Duraiswami ${ }^{\dagger}$ \\ Perceptual Interfaces and Reality Laboratory, \\ Institute for Advanced Computer Studies, \\ University of Maryland, College Park, Maryland, 20742. \\ ; Revised
}

(Received 


\begin{abstract}
A computational technique for the solution of problems of wave scattering from multiple spheres is developed. This technique, based on the T-matrix method, uses the theory for the translation and reexpansion of multipole solutions of the Helmholtz equation for fast and exact recursive computation of the matrix elements. The spheres can have prescribed radii, impedances, and locations. Results are validated by comparison with boundary element calculations, and by convergence analyses. The method is much faster than numerical methods based on discretization of space, or of the sphere surfaces. An even faster method is presented for the case when the spheres are aligned coaxially.
\end{abstract}

Short Title for Cover: Multiple Scattering from $N$ Spheres

Running Title: Multiple Scattering from $N$ Spheres

PACS numbers: $42.30 \mathrm{Fn}$

${ }^{*}$ URL: http://www. umiacs .umd.edu/users/gumerov; Electronic address: gumerov@umiacs.umd.edu

$\dagger$ Electronic address: ramani@umiacs.umd.edu; URL: http://www.umiacs.umd.edu/users/ramani 


\section{INTRODUCTION}

Numerous practical problems of acoustic and electromagnetic wave propagation require computation of the field scattered by multiple objects. Examples include the scattering of acoustic waves by objects (e.g., the scattering of sound by humans and the environment), light scattering by clouds and the environment, electromagnetic waves in composite materials and the human body, pressure waves in disperse systems (aerosols, emulsions, bubbly liquids), etc. Our interest is in the modeling the cues that arise due to scattering of sound

and light, and to use this information in simulating audio and video reality (Duda and Martens (1998), Duraiswami et al (2000)).

In many cases the scatterers are spheres, or can be modeled as such. Such modeling is convenient for parametrization of large problems, since each sphere can be characterized by a few quantities such as the coordinates of its center, its radius, and its impedance. This impedance will in general be a complex quantity, and characterizes the absorbing/reflecting properties of the body/surface. For example, we are exploring the modeling of the human head and body using two spheres representing respectively the head and the torso (Gumerov et al (2002), Algazi et al (2002)). In fluid mechanical problems, bubbles, droplets, or dust particles can be assumed spherical (Gumerov et al (1988), Duraiswami and Prosperetti $(1995))$.

We are interested in computing the solution of multiple scattering from $N$ spheres, with specified impedance boundary conditions at their surfaces. Numerical methods such as boundary-element methods (BEM), finite-element methods (FEM), or finite difference methods (FDM), are well known. Despite the relative advantages of these methods they all share a common deficiency related to the necessity of discretization of either the boundary surfaces, or of the complete space. Discretization introduces a characteristic size (or length scale) $l_{*}$ of the surface or spatial element. For accurate and stable computations the change of discretization length $l_{*}$ must not affect the results. This leads to a requirement that this size should be much smaller than the wavelength $\lambda$, i.e., $l_{*} \ll \lambda$. Practically this condition is $l_{*}<B \lambda$, where $B$ is some constant smaller than 1 . If computations are required for high frequencies (or short waves), this leads to very fine surface or spatial meshes. For example in computations of scattering of sound by human heads (Kahana (2001), Katz (2001)), for $20 \mathrm{kHz}$ sound in air in normal conditions the wavelength is $1.7 \mathrm{~cm}$, while the diameter of the 
typical human head is $D=17 \mathrm{~cm}$. For accuracy the length of a surface element should be, say, 6 times smaller than $1.7 \mathrm{~cm}$, i.e. 60 times smaller than the diameter of the head. This gives a 24000 element discretization of the head surface in case if triangular elements are used in BEM. Such discretizations require solution of very large linear systems with dense inter-influence matrices in the case of the BEM, and even larger, but sparse matrices in the case of the FEM/FDM. Solutions of these equations using direct or iterative methods are costly in terms of CPU time and memory.

Another approach to solve this problem is a semi-analytical method such as the T-matrix method (Waterman and Truell (1961), Peterson and Strom (1974), Varadan and Varadan (1980), Mischenko et al (1996), Koc and Chew (1998)). In this method, the scattering properties of a scatterer, and of the collection are characterized in terms of the so-called T-matrices that act on the coefficients of the local expansion of the incoming wave, centered at the scatterer, and transform them into the coefficients of an outgoing wave from the scatterer.

There are two basic challenges related to using the T-matrix method to solve multiple scattering problems. The first challenge is to obtain the solution for a single scatterer. In case of complex shapes and boundary conditions this solution can be obtained either numerically, or via analytical or semi-analytical techniques, based on decomposition of the original shape to objects of simpler geometry. The field scattered by sound-soft, sound-hard and elastic spheres placed in the field of a plane wave were considered in several papers, (e.g. Marnevskaya (1969, 1970), Huang and Gaunaurd (1995)). In the present paper we consider the practically important case of spherical objects with impedance boundary conditions in an arbitrary incident field (in particular that generated by a source). In this case a solution for a single scatterer in an arbitrary incident field can be obtained analytically.

The second challenge is related to computation of the T-matrices (or matrices of the translation coefficients for the Helmholtz equation). Several studies that are dedicated to scattering by two spheres (Marnevskaya (1969, 1970), Gaunaurd and Huang (1994), Gaunaurd et al (1995)) use representations of the translation coefficients via the Clebsch-Gordan coefficients or $3-j$ Wigner symbols. We should notice that while this method provides exact computation of T-matrix elements, it is time consuming, and, perhaps is practical only for a small number of spheres. Brunning and Lo (1971) considered the multiple scattering of electromagnetic waves by spheres, and pointed out that the computational work can be 
reduced by several orders of magnitude if the computation of the translation coefficients could be performed more efficiently, e.g. recursively. In their study they applied recursive computation of these coefficients for the case when the sphere centers are located on a line. This is always true for two spheres, and is a special case for three or more spheres. In the present paper we refer to this as the 'coaxial' case. A general three-dimensional case of acoustical scattering by $N$ objects using the T-matrix method was considered by Koc and Chew (1998). They proposed using a numerical evaluation of integral forms of the translation operators (Rokhlin, 1993) and the fast multipole method (FMM) applied to iterative solution of a large system of equations that appear in the T-matrix method.

In this paper we use a variant of the T-matrix technique that is specialized to the solution of the problem of multiple scattering from $N$ spheres arbitrary located in three-dimensional space. The distinguishing feature of our algorithm is utilization of a fast recursive procedure for exact computation of the T-matrix elements. We should note that the recurrence relations for three-dimensional scalar addition theorem for the Helmholtz equation were obtained by Chew (1992). These relations were derived for local-to-local (regular-to-regular) translations of the solutions of the Helmholtz equation, but can be extended to far-to-far and far-to-local translation. This was mentioned by Chew (1992) and details can be found in our study (Gumerov and Duraiswami, 2001) where also relations for arbitrary rotations and simplified expressions for coaxial translations are presented. The savings that would arise from use of fast multipole methods, can also be realized in our problem; though they are not considered here, as our focus is on the case of relatively small numbers of scatterers.

We have developed software implementing this technique. We compare the results of the computations with numerical and analytical solutions, and demonstrate the computational efficiency of our method in comparison with commercial BEM software. The results show that the developed method compares favorably in both accuracy and speed up of computations (in some cases by several orders of magnitude).

\section{STATEMENT OF THE PROBLEM}

Consider the problem of sound scattering by $N$ spheres with radii $a_{1}, \ldots, a_{N}$ situated in an infinite three dimensional space as shown in Fig. 1. The coordinates of the centers of the spheres are denoted as $\mathbf{r}_{p}^{\prime}=\left(x_{p}^{\prime}, y_{p}^{\prime}, z_{p}^{\prime}\right), p=1, \ldots, N$. The scattering problem in the 
frequency domain is reduced to solution of the Helmholtz equation for complex potential $\psi(\mathbf{r})$,

$$
\nabla^{2} \psi+k^{2} \psi=0
$$

with the following general impedance boundary conditions on the surface $S_{p}$ of the $p$ th sphere:

$$
\left.\left(\frac{\partial \psi}{\partial n}+i \sigma_{p} \psi\right)\right|_{S_{p}}=0, \quad p=1, \ldots, N,
$$

where $k$ is the wavenumber and $\sigma_{p}$ are complex admittances, and $i=\sqrt{-1}$. In the particular case of sound-hard surfaces $\left(\sigma_{p}=0\right)$ we have the Neumann boundary conditions,

$$
\partial \psi /\left.\partial n\right|_{S_{p}}=0
$$

and in the case of sound-soft surfaces $\left(\sigma_{p}=\infty\right)$ we have the Dirichlet boundary conditions,

$$
\left.\psi\right|_{S_{p}}=0
$$

Usually the potential is represented in the form:

$$
\psi(\mathbf{r})=\psi_{\text {in }}(\mathbf{r})+\psi_{\text {scat }}(\mathbf{r})
$$

where $\psi_{\text {scat }}(\mathbf{r})$ is the potential of the scattered field. Far from the region occupied by spheres the scattered field should satisfy the Sommerfeld radiation condition:

$$
\lim _{r \rightarrow \infty} r\left(\frac{\partial \psi_{\text {scat }}}{\partial r}-i k \psi_{\text {scat }}\right)=0 .
$$

\section{SOLUTION USING MULTIPOLE TRANSLATION AND REEXPANSION}

\section{A. Decomposition of the scattered field}

Due to the linearity of the problem the scattered field can be represented in the form

$$
\psi_{\text {scat }}(\mathbf{r})=\sum_{p=1}^{N} \psi_{p}(\mathbf{r}),
$$

where $\psi_{p}(\mathbf{r})$ can loosely be thought of as the change in the scattered field introduced by the $p$ th sphere, though of course it contains the influence of all the spheres. More precisely, each potential $\psi_{p}(\mathbf{r})$ is regular outside the $p$ th sphere and satisfies the Sommerfeld radiation condition

$$
\lim _{r \rightarrow \infty} r\left(\frac{\partial \psi_{p}}{\partial r}-i k \psi_{p}\right)=0, \quad p=1, \ldots, N .
$$




\section{B. Multipole expansion}

Let us introduce $N$ reference frames connected with the center of each sphere. In spherical polar coordinates $\mathbf{r}-\mathbf{r}_{p}^{\prime}=\mathbf{r}_{p}=\left(r_{p}, \theta_{p}, \varphi_{p}\right)$, the solution of the Helmholtz equation that satisfies the radiation condition (8) can be represented in the form

$$
\psi_{p}(\mathbf{r})=\sum_{n=0}^{\infty} \sum_{m=-n}^{n} A_{n}^{(p) m} S_{n}^{m}\left(\mathbf{r}_{p}\right), \quad p=1, \ldots, N
$$

Here $A_{n}^{(p) m}$ are coefficients multiplying the $S_{n}^{m}(\mathbf{r})$, which are multipoles of order $n$ and degree $m$, given by

$$
S_{n}^{m}\left(\mathbf{r}_{p}\right)=h_{n}\left(k r_{p}\right) Y_{n}^{m}\left(\theta_{p}, \varphi_{p}\right), \quad p=1, \ldots, N
$$

Here $h_{n}(k r)$ are spherical Hankel functions of the 1st kind that satisfy the Sommerfeld condition, and $Y_{n}^{m}(\theta, \phi)$ are orthonormal spherical harmonics, which also can be represented in the form

$$
Y_{n}^{m}(\theta, \varphi)=(-1)^{m} \sqrt{\frac{2 n+1}{4 \pi} \frac{(n-|m|) !}{(n+|m|) !}} P_{n}^{|m|}(\cos \theta) e^{i m \varphi}
$$

where $P_{n}^{m}(\mu)$ are the associated Legendre functions.

The problem now is to determine coefficients $A_{n}^{(p) m}$ so that the complete potential

$$
\psi(\mathbf{r})=\psi_{i n}(\mathbf{r})+\sum_{p=1}^{N} \sum_{n=0}^{\infty} \sum_{m=-n}^{n} A_{n}^{(p) m} S_{n}^{m}\left(\mathbf{r}_{p}\right)
$$

satisfies all boundary conditions on the surface of all spheres.

\section{Multipole reexpansion}

To solve this problem let us consider the $q$ th sphere. Near the center of this sphere, $\mathbf{r}=\mathbf{r}_{q}^{\prime}$, all the potentials $\psi_{p}(\mathbf{r}), p \neq q$, are regular. Accordingly the singular solutions (multipoles $\left.S_{n}^{m}\left(\mathbf{r}_{p}\right), p \neq q\right)$ can be then re-expanded into a series in terms of regular elementary solutions near the $q$ th sphere, $\left|\mathbf{r}_{q}\right| \leqslant\left|\mathbf{r}_{p}^{\prime}-\mathbf{r}_{q}^{\prime}\right|$ using the following expression

$$
S_{n}^{m}\left(\mathbf{r}_{p}\right)=\sum_{l=0}^{\infty} \sum_{s=-l}^{l}(S \mid R)_{l n}^{s m}\left(\mathbf{r}_{p q}^{\prime}\right) R_{l}^{s}\left(\mathbf{r}_{q}\right), \quad p, q=1, \ldots, N, \quad p \neq q
$$


Here $R_{n}^{m}\left(\mathbf{r}_{q}\right)$ are regular elementary solutions of the Helmholtz equation in spherical coordinates connected with the $q$ th sphere:

$$
R_{n}^{m}\left(\mathbf{r}_{q}\right)=j_{n}\left(k r_{q}\right) Y_{n}^{m}\left(\theta_{q}, \varphi_{q}\right), \quad p=1, \ldots, N
$$

where $j_{n}(k r)$ are spherical Bessel functions of the first kind. The coefficients $(S \mid R)_{l n}^{s m}\left(\mathbf{r}_{p q}^{\prime}\right)$ are the translation reexpansion coefficients, and depend on the relative locations of the $p$ th and $q$ th spheres, $\mathbf{r}_{p q}^{\prime}$. Since $\mathbf{r}=\mathbf{r}_{p}+\mathbf{r}_{p}^{\prime}=\mathbf{r}_{q}+\mathbf{r}_{q}^{\prime}$, we have

$$
\mathbf{r}_{p}=\mathbf{r}_{q}+\mathbf{r}_{p q}^{\prime}, \quad \mathbf{r}_{p q}^{\prime}=\mathbf{r}_{q}^{\prime}-\mathbf{r}_{p}^{\prime}=\mathbf{r}_{p}-\mathbf{r}_{q},
$$

where $\mathbf{r}_{p q}^{\prime}$ is the vector directed from the center of the $p$ th sphere to the center of the $q$ th sphere. Aspects of the multipole reexpansion coefficients, their properties, and methods for their efficient computation are considered in Chew (1992) and Gumerov and Duraiswami (2001).

Near this center of expansion the incident field can be also represented using a similar series, the radius of convergence for which is not smaller than the radius of the $q$ th sphere:

$$
\psi_{i n}(\mathbf{r})=\sum_{l=0}^{\infty} \sum_{s=-l}^{l} C_{l}^{(i n) s}\left(\mathbf{r}_{q}^{\prime}\right) R_{l}^{s}\left(\mathbf{r}_{q}\right) .
$$

Substituting the local expansions from Eqs. (13) and (16) into Eq. (12) we obtain the following representation of the field near $\mathbf{r}=\mathbf{r}_{q}^{\prime}$

$$
\begin{aligned}
\psi(\mathbf{r}) & =\sum_{l=0}^{\infty} \sum_{s=-l}^{l} C_{l}^{(i n) s}\left(\mathbf{r}_{q}^{\prime}\right) R_{l}^{s}\left(\mathbf{r}_{q}\right)+\sum_{n=0}^{\infty} \sum_{m=-n}^{n} A_{n}^{(q) m} S_{n}^{m}\left(\mathbf{r}_{q}\right) \\
& +\sum_{\substack{p=1 \\
p \neq q}}^{n} \sum_{n=0}^{\infty} \sum_{m=-n}^{n} A_{n}^{(p) m} \sum_{l=0}^{\infty} \sum_{s=-l}^{l}(S \mid R)_{l n}^{s m}\left(\mathbf{r}_{p q}^{\prime}\right) R_{l}^{s}\left(\mathbf{r}_{q}\right) .
\end{aligned}
$$

Here the incident field is also expanded in terms of the regular functions centered at $\mathbf{r}_{q}$, the singular multipole expansion around $\mathbf{r}_{q}$ is kept as is, while the other multipole expansions are converted to local expansions in terms of regular functions around $\mathbf{r}_{q}$.

Let us change the order of summation in the latter term in Eq. (17) and substitute Eqs. (10) and (14) for $S_{n}^{m}$ and $R_{n}^{m}$. This expression can then be rewritten as

$$
\begin{aligned}
\psi(\mathbf{r}) & =\sum_{l=0}^{\infty} \sum_{s=-l}^{l}\left[B_{l}^{(q) s} j_{l}\left(k r_{q}\right)+A_{l}^{(q) s} h_{l}\left(k r_{q}\right)\right] Y_{l}^{s}\left(\theta_{q}, \varphi_{q}\right), \\
B_{l}^{(q) s}\left(\mathbf{r}_{1}^{\prime}, \ldots, \mathbf{r}_{N}^{\prime}\right) & =C_{l}^{(i n) s}\left(\mathbf{r}_{q}^{\prime}\right)+\sum_{\substack{p=1 \\
p \neq q}}^{N} \sum_{n=0}^{\infty} \sum_{m=-n}^{n}(S \mid R)_{l n}^{s m}\left(\mathbf{r}_{p q}^{\prime}\right) A_{n}^{(p) m}
\end{aligned}
$$




\section{Boundary conditions}

From these equations we have the boundary values of $\psi$ and $\partial \psi / \partial n$ on the surface of the $q$ th sphere as

$$
\begin{aligned}
\left.\psi\right|_{S_{q}} & =\sum_{l=0}^{\infty} \sum_{s=-l}^{l}\left[B_{l}^{(q) s} j_{l}\left(k a_{q}\right)+A_{l}^{(q) s} h_{l}\left(k a_{q}\right)\right] Y_{l}^{s}\left(\theta_{q}, \varphi_{q}\right), \\
\left.\frac{\partial \psi}{\partial n}\right|_{S_{q}} & =k \sum_{l=0}^{\infty} \sum_{s=-l}^{l}\left[B_{l}^{(q) s} j_{l}^{\prime}\left(k a_{q}\right)+A_{l}^{(q) s} h_{l}^{\prime}\left(k a_{q}\right)\right] Y_{l}^{s}\left(\theta_{q}, \varphi_{q}\right),
\end{aligned}
$$

To obtain the coefficients, we must satisfy the boundary condition Eq. (2), which on the surface of the $q$ th sphere, leads to

$$
\sum_{l=0}^{\infty} \sum_{s=-l}^{l}\left\{B_{l}^{(q) s}\left[k j_{l}^{\prime}\left(k a_{q}\right)+i \sigma_{q} j_{l}\left(k a_{q}\right)\right]+A_{l}^{(q) s}\left[k h_{l}^{\prime}\left(k a_{q}\right)+i \sigma_{q} h_{l}\left(k a_{q}\right)\right]\right\} Y_{l}^{s}\left(\theta_{q}, \varphi_{q}\right)=0 .
$$

Orthogonality of the surface harmonics yields:

$$
B_{l}^{(q) s}\left[k j_{l}^{\prime}\left(k a_{q}\right)+i \sigma_{q} j_{l}\left(k a_{q}\right)\right]=-A_{l}^{(q) s}\left[k h_{l}^{\prime}\left(k a_{q}\right)+i \sigma_{q} h_{l}\left(k a_{q}\right)\right],
$$

where $l=0,1, \ldots, \quad s=-l, \ldots, l$. Note that the boundary values of $\left.\psi\right|_{S_{q}}$ and $\partial \psi /\left.\partial n\right|_{S_{q}}$ can be expressed in terms of the coefficients $A_{l}^{(q) s}$, since

$$
B_{l}^{(q) s}=-A_{l}^{(q) s} \frac{k h_{l}^{\prime}\left(k a_{q}\right)+i \sigma_{q} h_{l}\left(k a_{q}\right)}{k j_{l}^{\prime}\left(k a_{q}\right)+i \sigma_{q} j_{l}\left(k a_{q}\right)}, \quad l=0,1, \ldots, \quad s=-l, \ldots, l .
$$

and Eqs. (20) and (21) yield

$$
\begin{aligned}
\left.\psi\right|_{S_{q}} & =\sum_{l=0}^{\infty} \sum_{s=-l}^{l}\left[h_{l}\left(k a_{q}\right)-j_{l}\left(k a_{q}\right) \frac{k h_{l}^{\prime}\left(k a_{q}\right)+i \sigma_{q} h_{l}\left(k a_{q}\right)}{k j_{l}^{\prime}\left(k a_{q}\right)+i \sigma_{q} j_{l}\left(k a_{q}\right)}\right] A_{l}^{(q) s} Y_{l}^{s}\left(\theta_{q}, \varphi_{q}\right), \\
\left.\frac{\partial \psi}{\partial n}\right|_{S_{q}} & =k \sum_{l=0}^{\infty} \sum_{s=-l}^{l}\left[h_{l}^{\prime}\left(k a_{q}\right)-j_{l}^{\prime}\left(k a_{q}\right) \frac{k h_{l}^{\prime}\left(k a_{q}\right)+i \sigma_{q} h_{l}\left(k a_{q}\right)}{k j_{l}^{\prime}\left(k a_{q}\right)+i \sigma_{q} j_{l}\left(k a_{q}\right)}\right] A_{l}^{(q) s} Y_{l}^{s}\left(\theta_{q}, \varphi_{q}\right) .
\end{aligned}
$$

These relations can be also rewritten in a compact form using the Wronskian for the spherical Bessel functions,

$$
W\left\{j_{l}(k a), h_{l}(k a)\right\}=j_{l}(k a) h_{l}^{\prime}(k a)-j_{l}^{\prime}(k a) h_{l}(k a)=i(k a)^{-2}
$$

as

$$
\begin{aligned}
\left.\psi\right|_{S_{q}} & =\frac{1}{i k a_{q}^{2}} \sum_{l=0}^{\infty} \sum_{s=-l}^{l} \frac{A_{l}^{(q) s} Y_{l}^{s}\left(\theta_{q}, \varphi_{q}\right)}{k j_{l}^{\prime}\left(k a_{q}\right)+i \sigma_{q} j_{l}\left(k a_{q}\right)}, \\
\left.\frac{\partial \psi}{\partial n}\right|_{S_{q}} & =-\frac{\sigma_{q}}{k a_{q}^{2}} \sum_{l=0}^{\infty} \sum_{s=-l}^{l} \frac{A_{l}^{(q) s} Y_{l}^{s}\left(\theta_{q}, \varphi_{q}\right)}{k j_{l}^{\prime}\left(k a_{q}\right)+i \sigma_{q} j_{l}\left(k a_{q}\right)}=-\left.i \sigma_{q} \psi\right|_{S_{q}} .
\end{aligned}
$$


For the particular case of a sound-hard spheres $\left(\sigma_{q}=0\right)$ this gives

$$
\left.\psi\right|_{S_{q}}=\frac{1}{i k^{2} a_{q}^{2}} \sum_{l=0}^{\infty} \sum_{s=-l}^{l} \frac{A_{l}^{(q) s} Y_{l}^{s}\left(\theta_{q}, \varphi_{q}\right)}{j_{l}^{\prime}\left(k a_{q}\right)},\left.\quad \frac{\partial \psi}{\partial n}\right|_{S_{q}}=0
$$

while for sound-soft spheres $\left(\sigma_{q}=\infty\right)$ we have

$$
\left.\psi\right|_{S_{q}}=0,\left.\quad \frac{\partial \psi}{\partial n}\right|_{S_{q}}=\frac{i}{k a_{q}^{2}} \sum_{l=0}^{\infty} \sum_{s=-l}^{l} \frac{A_{l}^{(q) s} Y_{l}^{s}\left(\theta_{q}, \varphi_{q}\right)}{j_{l}\left(k a_{q}\right)}
$$

\section{E. Matrix representation}

To determine the boundary values of the potential and/or its normal derivative and obtain a spatial distribution according to Eq. (12) we need to determine the coefficients $A_{l}^{(q) s}$ in Eqs. (23) and (19), which are valid for any sphere, $q=1, \ldots, N$. These equations form a linear system that may be represented in standard matrix-vector form.

First, we note that coefficients of expansions to spherical harmonics, such as $A_{n}^{m}, n=$ $0,1,2, \ldots ; m=-n, \ldots, n$, can be stacked into one column vector, e.g.

$$
\mathbf{A}=\left(A_{0}^{0}, A_{1}^{-1}, A_{1}^{0}, A_{1}^{1}, A_{2}^{-2}, A_{2}^{-1}, A_{2}^{0}, A_{2}^{1}, A_{2}^{2}, \ldots\right)^{T}
$$

where the superscript $T$ denotes the transpose. In this representation the elements of the vector $\mathbf{A}$ are related to coefficients $A_{n}^{m}$ by

$$
A_{t}=A_{n}^{m}, \quad t=(n+1)^{2}-(n-m), \quad n=0,1,2, \ldots ; \quad m=-n, \ldots, n ; \quad t=1,2, \ldots
$$

The same reduction in dimension can be applied to the reexpansion coefficients $(S \mid R)_{l n}^{s m}$. The coefficients can then be stacked in a 2-dimensional matrix as

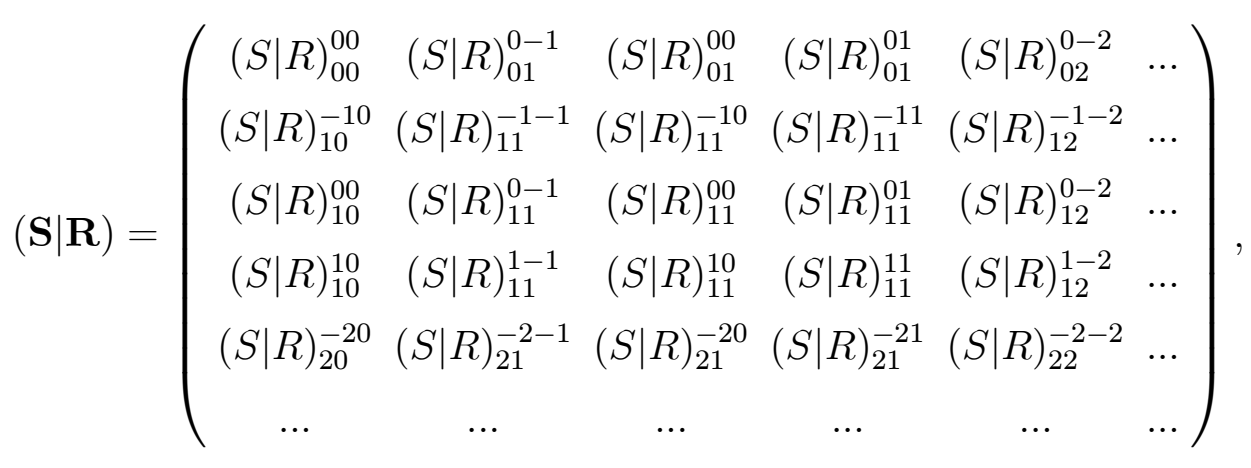


with the following correspondence of the matrix elements and coefficients:

$$
\begin{aligned}
(S \mid R)_{r t} & =(S \mid R)_{l n}^{s m}, \quad r=(l+1)^{2}-(l-s), \quad t=(n+1)^{2}-(n-m) \\
l, n & =0,1,2, \ldots ; \quad m=-n, \ldots, n ; \quad s=-l, \ldots, l .
\end{aligned}
$$

Using this representation we introduce the following vectors and matrices

$$
\mathbf{A}^{(q)}=\left\{A_{t}^{(q)}\right\}^{T}, \quad \mathbf{D}^{(q)}=\left\{D_{t}^{(q)}\right\}^{T}, \quad \mathbf{L}^{(q p)}=\left\{L_{r t}^{(q p)}\right\}, \quad q=1, \ldots, N, \quad p=1, \ldots, N .
$$

where

$$
\begin{aligned}
A_{t}^{(q)} & =A_{n}^{(q) m}, \quad D_{t}^{(q)}=-\frac{k j_{n}^{\prime}\left(k a_{q}\right)+i \sigma_{q} j_{n}\left(k a_{q}\right)}{k h_{n}^{\prime}\left(k a_{q}\right)+i \sigma_{q} h_{n}\left(k a_{q}\right)} C_{n}^{(i n) m}\left(\mathbf{r}_{q}^{\prime}\right), \\
L_{r t}^{(q p)} & =\frac{k j_{l}^{\prime}\left(k a_{q}\right)+i \sigma_{q} j_{l}\left(k a_{q}\right)}{k h_{l}^{\prime}\left(k a_{q}\right)+i \sigma_{q} h_{l}\left(k a_{q}\right)}(S \mid R)_{l n}^{s m}\left(\mathbf{r}_{p q}^{\prime}\right), \quad \text { for } p \neq q, \quad L_{r t}^{(q q)}=\delta_{r t}, \\
t & =(n+1)^{2}-(n-m), \quad n=0,1,2, \ldots ; \quad m=-n, \ldots, n ; \\
r & =(l+1)^{2}-(l-s), \quad l=0,1,2, \ldots ; \quad s=-l, \ldots, l ; \quad q=1, \ldots, N, \quad p=1, \ldots, N,
\end{aligned}
$$

where $\delta_{r t}$ is the Kronecker delta, $\delta_{r t}=0$ for $r \neq t$ and $\delta_{r r}=1$.

Equations (23) and (19) then can be represented in the form

$$
\sum_{p=1}^{N} \mathbf{L}^{(q p)} \mathbf{A}^{(p)}=\mathbf{D}^{(q)}, \quad q=1, \ldots, N,
$$

or as a single equation

$$
\mathbf{L A}=\mathbf{D},
$$

where the matrices and vectors are

$$
\mathbf{L}=\left(\begin{array}{cccc}
\mathbf{L}^{(11)} & \mathbf{L}^{(12)} & \ldots & \mathbf{L}^{(1 N)} \\
\mathbf{L}^{(21)} & \mathbf{L}^{(22)} & \ldots & \mathbf{L}^{(2 N)} \\
\ldots & \ldots & \ldots & \ldots \\
\mathbf{L}^{(N 1)} & \mathbf{L}^{(N 2)} & \ldots & \mathbf{L}^{(N N)}
\end{array}\right), \quad \mathbf{A}=\left(\begin{array}{c}
\mathbf{A}^{(1)} \\
\mathbf{A}^{(2)} \\
\ldots \\
\mathbf{A}^{(N)}
\end{array}\right), \quad \mathbf{D}=\left(\begin{array}{c}
\mathbf{D}^{(1)} \\
\mathbf{D}^{(2)} \\
\ldots \\
\mathbf{D}^{(N)}
\end{array}\right)
$$

This linear system can be solved numerically using standard routines, such as LUdecomposition. The block-structure can also be exploited using block-oriented solvers, though we do not pursue this here. 
An important issue is the truncation of the infinite series and corresponding truncation of the associated matrices. A first way to truncate the series is to truncate the outer sum at a fixed number $M$ in each expansion, and is the approach taken here. This number is selected via a heuristic based on the magnitude of the smallest retained term. In this case $n=0,1, \ldots, M, m=-n, \ldots, n$, then the length of each vector $\mathbf{A}^{(p)}$ and $\mathbf{D}^{(p)}$ will be $(M+1)^{2}$, and the size of each sub-matrix $\mathbf{L}^{(q p)}$ will be $(M+1)^{2} \times(M+1)^{2}$, the size of the total vectors A and $\mathbf{D}$ will be $N(M+1)^{2}$ and the size of the total matrix $\mathbf{L}$ will be $N(M+1)^{2} \times N(M+1)^{2}$.

Several ideas could be employed to improve this heuristic. For example, the use of fast multipole methods could lead to accelerated schemes to solve these equations iteratively (Koc and Chew, 1998). Further, the expressions used to select the truncation of the series could be selected in a more rigorous manner. However, as will be seen below, even with a fixed size of $M$ we are able to solve large problems using conventional techniques.

\section{F. Computation of multipole reexpansion coefficients}

The $(S \mid R)$-multipole translation coefficients can be computed in different ways including via numerical evaluation of integral representations, or using the Clebsch-Gordan or Wigner 3- $j$ symbols (e.g. see Epton and Dembart (1994), Koc et al (1999)). For fast, stable, exact and efficient computations of the entire truncated matrix of the reexpansion coefficients we used a method based on Chew (1992) recurrence relations and symmetries, who derived them first for the $(R \mid R)$-translation coefficients (translation of regular-to-regular elementary solution, see Eq. (14)). Fortunately (since the singular and regular elementary solutions of the Helmholtz equation satisfy the same recurrence relations) these relations hold for $(S \mid R)$-multipole translation coefficients also, but with different initial values for recursive computations (44). This was also pointed out by Chew (1992). Note that for other equations (e.g. for the Laplace equation) the singular and regular elementary solutions may not satisfy the same recursions. In Gumerov and Duraiswami (2001) proofs and details may be found. We only provide necessary relations and initial values for using the recurrence procedures here.

All the $(S \mid R)_{l n}^{s m}\left(\mathbf{r}_{p q}^{\prime}\right)$ translation reexpansion coefficients can be computed in the following way. First, we compute the so-called "sectorial coefficients" of type $(S \mid R)_{l|m|}^{s m}$ and $(S \mid R)_{|s| n}^{s m}$ 
using the following relations:

$$
\begin{aligned}
b_{m+1}^{-m-1}(S \mid R)_{l, m+1}^{s, m+1} & =b_{l}^{-s}(S \mid R)_{l-1, m}^{s-1, m}-b_{l+1}^{s-1}(S \mid R)_{l+1, m}^{s-1, m} \\
b_{m+1}^{-m-1}(S \mid R)_{l, m+1}^{s,-m-1} & =b_{l}^{s}(S \mid R)_{l-1, m}^{s+1,-m}-b_{l+1}^{-s-1}(S \mid R)_{l+1, m}^{s+1,-m} \\
l & =0,1, \ldots \quad s=-l, \ldots, l, \quad m=0,1,2, \ldots
\end{aligned}
$$

where

$$
b_{n}^{m}=\left\{\begin{array}{cc}
\sqrt{\frac{(n-m-1)(n-m)}{(2 n-1)(2 n+1)}}, & 0 \leqslant m \leqslant n, \\
-\sqrt{\frac{(n-m-1)(n-m)}{(2 n-1)(2 n+1)}}, & -n \leqslant m<0, \\
0, & |m|>n,
\end{array}\right.
$$

and the recurrence process starts with

$$
(S \mid R)_{l 0}^{s 0}\left(\mathbf{r}_{p q}^{\prime}\right)=\sqrt{(4 \pi)}(-1)^{l} S_{l}^{-s}\left(\mathbf{r}_{p q}^{\prime}\right), \quad(S \mid R)_{0 n}^{0 m}\left(\mathbf{r}_{p q}^{\prime}\right)=\sqrt{(4 \pi)} S_{n}^{m}\left(\mathbf{r}_{p q}^{\prime}\right)
$$

Due to the symmetry relation

$$
(S \mid R)_{|m| l}^{-m,-s}=(-1)^{l+m}(S \mid R)_{l|m|}^{s m}, \quad l=0,1,2, \ldots, \quad s=-l, \ldots, l, \quad m=-n, \ldots, n
$$

all of the sectorial coefficients $(S \mid R)_{|s| n}^{s m}$ can be obtained from the coefficients $(S \mid R)_{l|m|}^{s m}$.

Once the sectorial coefficients are computed all other coefficients can be derived from them using the following recurrence relation, which does not change the degrees $s, m$ of the reexpansion coefficients:

$$
\begin{aligned}
a_{n-1}^{m}(S \mid R)_{l, n-1}^{s m}-a_{n}^{m}(S \mid R)_{l, n+1}^{s m} & =a_{l}^{s}(S \mid R)_{l+1, n}^{s m}-a_{l-1}^{s}(S \mid R)_{l-1, n}^{s m}, \\
l, n & =0,1, \ldots \quad s=-l, \ldots, l, \quad m=-n, \ldots, n
\end{aligned}
$$

where

$$
a_{n}^{m}=\left\{\begin{array}{c}
\sqrt{\frac{(n+1+|m|)(n+1-|m|)}{(2 n+1)(2 n+3)}}, \quad n \geqslant|m|, \\
0, \quad|m|>n .
\end{array}\right.
$$

Due to the symmetry

$$
(S \mid R)_{l n}^{s m}=(-1)^{n+l}(S \mid R)_{n l}^{-m,-s}, \quad l, n=0,1, \ldots \quad s=-l, \ldots, l, \quad m=-n, \ldots, n .
$$

only those coefficients with $l \geqslant n$ need be computed using recurrence relations. 
In addition, the $(S \mid R)$ coefficients for any pair of spheres $p$ and $q$ need be computed only for the vector $\mathbf{r}_{p q}^{\prime}$, since for the opposite directed vector we have the symmetry

$$
(S \mid R)_{l n}^{s m}\left(\mathbf{r}_{q p}^{\prime}\right)=(-1)^{l+n}(S \mid R)_{l n}^{s m}\left(\mathbf{r}_{p q}^{\prime}\right), \quad l, n=0,1, \ldots, \quad m=-n, \ldots, n
$$

Software based on this algorithm was developed and entitled MultisphereHelmholtz. Results of tests using this software are presented and discussed in section $\mathrm{V}$.

\section{COAXIAL SPHERES}

The case of two spheres is interesting, since on the one hand the scattered fields due to the spheres interact with each other (multiple scattering), while on the other, the interaction is still simple enough that it can be investigated in detail and understood more intuitively than the general case of $N$ spheres. Additionally, in this case, the computation of the reexpansion matrices can be simplified via a proper selection of the reference frames. Indeed, for two spheres we can introduce a reference frame which has its $z$ axis directed from the center of one sphere to the center of the other sphere. Since the reexpansion coefficients depend only on the relative locations of the spheres, for this frame orientation, there will be no angular dependence for the reexpansion coefficients. This does not require the incident fields to be symmetric with respect to this axis. The same statement holds for the case when there are $N$ spheres arranged along a line, taken to be the $z$ axis. In these particular cases, the general reexpansion formula Eq. (13) simplifies to

$$
S_{n}^{m}\left(\mathbf{r}_{p}\right)=\sum_{l=|m|}^{\infty}(S \mid R)_{l n}^{m m}\left(\mathbf{r}_{p q}^{\prime}\right) R_{l}^{m}\left(\mathbf{r}_{q}\right), \quad p=1, \ldots, N, \quad p \neq q .
$$

The coefficients

$$
(S \mid R)_{l n}^{m}\left(r_{p q}^{\prime}\right)=(S \mid R)_{l n}^{m m}\left(\mathbf{r}_{p q}^{\prime}\right), \quad l, n=0,1, \ldots, \quad m=-n, \ldots, n
$$

satisfy general recurrence relations and can be computed using the general algorithm we have developed. However, there are simpler relations that take advantage of the co-axiality of the spheres, while resulting in substantially lower dimensional matrices.

Note that the sign of coefficients $(S \mid R)_{l n}^{m}\left(r_{p q}^{\prime}\right)$ depends on the direction of the vector $\mathbf{r}_{p q}^{\prime}$. To be definite, we use the convention that $r_{p q}^{\prime}$ corresponds to $\mathbf{r}_{p q}^{\prime}$ and $r_{q p}^{\prime}$ corresponds to 
$\mathbf{r}_{q p}^{\prime}=-\mathbf{r}_{p q}^{\prime}$. Since $(S \mid R)_{l n}^{m m}\left(\mathbf{r}_{p q}^{\prime}\right)=(-1)^{l+n}(S \mid R)_{l n}^{m m}\left(-\mathbf{r}_{p q}^{\prime}\right)=(-1)^{l+n}(S \mid R)_{l n}^{m m}\left(\mathbf{r}_{q p}^{\prime}\right)$, we will have

$$
(S \mid R)_{l n}^{m}\left(r_{p q}^{\prime}\right)=(-1)^{l+n}(S \mid R)_{l n}^{m}\left(r_{q p}^{\prime}\right), \quad l, n=0,1, \ldots, \quad m=-n, \ldots, n
$$

\section{A. Matrix representation}

According to Eq. (50) harmonics of each order $m$ can be considered independently. Equations (19) and (23) can be rewritten in the form:

$$
\begin{array}{r}
\frac{k j_{l}^{\prime}\left(k a_{q}\right)+i \sigma_{q} j_{l}\left(k a_{q}\right)}{k h_{l}^{\prime}\left(k a_{q}\right)+i \sigma_{q} h_{l}\left(k a_{q}\right)} \sum_{\substack{p=1 \\
p \neq q}}^{N} \sum_{n=|m|}^{\infty}(S \mid R)_{l n}^{m}\left(r_{p q}^{\prime}\right) A_{n}^{(p) m}+A_{l}^{(q) m}=D_{l}^{(q) m} \\
m=0, \pm 1, \pm 2, \ldots, \quad l=|m|,|m|+1, \ldots, \quad q=1, \ldots, N
\end{array}
$$

where

$$
D_{l}^{(q) m}=-\frac{k j_{l}^{\prime}\left(k a_{q}\right)+i \sigma_{q} j_{l}\left(k a_{q}\right)}{k h_{l}^{\prime}\left(k a_{q}\right)+i \sigma_{q} h_{l}\left(k a_{q}\right)} C_{l}^{(i n) m}\left(\mathbf{r}_{q}^{\prime}\right) .
$$

This linear system can be represented in the following form

$$
\sum_{p=1}^{N} \mathbf{L}^{(q p) m} \mathbf{A}^{(p) m}=\mathbf{D}^{(q) m}, \quad m=0, \pm 1, \pm 2, \ldots, \quad q=1, \ldots, N
$$

where the vectors $\mathbf{A}^{(q) m}$ and $\mathbf{D}^{(q) m}$ and matrices $\mathbf{L}^{(q p) m}$ are stacked as follows

$$
\begin{aligned}
\mathbf{A}^{(q)} & =\left\{A_{n}^{(q) m}\right\}^{T}, \quad \mathbf{D}^{(q) m}=\left\{D_{n}^{(q) m}\right\}^{T}, \quad \mathbf{L}^{(q p) m}=\left\{L_{l n}^{(q p) m}\right\} \\
& q=1, \ldots, N, \quad p=1, \ldots, N, \quad m=0, \pm 1, \pm 2, \ldots, \quad l, n=|m|,|m|+1, \ldots
\end{aligned}
$$

with the individual matrix elements given by

$$
L_{l n}^{(q p) m}=\frac{k j_{l}^{\prime}\left(k a_{q}\right)+i \sigma_{q} j_{l}\left(k a_{q}\right)}{k h_{l}^{\prime}\left(k a_{q}\right)+i \sigma_{q} h_{l}\left(k a_{q}\right)}(S \mid R)_{l n}^{m}\left(r_{p q}^{\prime}\right), \quad \text { for } p \neq q, \quad L_{l n}^{(q q) m}=\delta_{l n} .
$$

Since all equations can be considered separately for each $m$, the linear system Eq. (55) can be written as

$$
\mathbf{L}^{m} \mathbf{A}^{m}=\mathbf{D}^{m}, \quad m=0, \pm 1, \pm 2, \ldots
$$


where

$$
\mathbf{L}^{m}=\left(\begin{array}{cccc}
\mathbf{L}^{(11) m} & \mathbf{L}^{(12) m} & \ldots & \mathbf{L}^{(1 N) m} \\
\mathbf{L}^{(21) m} & \mathbf{L}^{(22) m} & \ldots & \mathbf{L}^{(2 N) m} \\
\ldots & \ldots & \ldots & \ldots \\
\mathbf{L}^{(N 1) m} & \mathbf{L}^{(N 2) m} & \ldots & \mathbf{L}^{(N N) m}
\end{array}\right), \quad \mathbf{A}^{m}=\left(\begin{array}{c}
\mathbf{A}^{(1) m} \\
\mathbf{A}^{(2) m} \\
\ldots \\
\mathbf{A}^{(N) m}
\end{array}\right), \quad \mathbf{D}^{m}=\left(\begin{array}{c}
\mathbf{D}^{(1) m} \\
\mathbf{D}^{(2) m} \\
\ldots \\
\mathbf{D}^{(N) m}
\end{array}\right)
$$

As in the general case considered above, the infinite series and matrices need to be truncated for numerical computation. If we limit ourselves to the first $M$ modes for each expansion of spherical harmonics, so that $m=0, \pm 1, \ldots, \pm M, n=|m|,|m|+1, \ldots, M$, then the length of each vector $\mathbf{A}^{(p) m}$ and $\mathbf{D}^{(p) m}$ is $M+1-|m|$, the dimensions of each matrix $\mathbf{L}^{(q p) m}$ is $(M+1-|m|) \times(M+1-|m|)$, the size of the total vectors $\mathbf{A}^{m}$ and $\mathbf{D}^{m}$ are $N(M+1-|m|)$, and the size of the total matrix $\mathbf{L}^{m}$ is $N(M+1-|m|) \times N(M+1-|m|)$. The problem then is reduced to solution of $2 M+1$ independent linear systems for each $m$. Note that the coaxial, or diagonal, translation coefficients $(S \mid R)_{l n}^{m}\left(r_{p q}^{\prime}\right)$ are symmetrical with respect to the sign of the degree $m,(S \mid R)_{l n}^{m}\left(r_{p q}^{\prime}\right)=(S \mid R)_{l n}^{-m}\left(r_{p q}^{\prime}\right)$ (see Gumerov and Duraiswami (2001)). Therefore the matrices $\mathbf{L}^{m}$ are also symmetrical (see Eq. (57))

$$
\mathbf{L}^{m}=\mathbf{L}^{-m}, \quad m=0,1,2, \ldots
$$

and can be computed only for non-negative $m$. At the same time the right-hand side vector $\mathbf{D}^{m}$, generally speaking, does not coincide with $\mathbf{D}^{-m}$, so that the solution $\mathbf{A}^{m}$ can be different from $\mathbf{A}^{-m}$.

Let us compare the number of operations required for determination of all expansion coefficients $A_{n}^{(q) m}, m=0, \pm 1, \ldots, \pm M, n=|m|,|m|+1, \ldots, M, q=1, \ldots, N$, using the general algorithm and using the algorithm for coaxial spheres. Assuming that a standard solver requires $C K^{3}$ operations to solve a linear system with matrix of dimension $K \times K$, where $C$ is some constant, we can find solution using general algorithm with

$$
N_{\text {operations }}^{(\text {general })}=C N^{3}(M+1)^{6}
$$

operations. Using the algorithm for coaxial spheres we will spend $C N^{3}(M+1-|m|)^{3}$ operations to obtain $A_{n}^{(q) m}$ for each $m=0, \pm 1, \ldots,, \pm M$. The total number of operations will 
be therefore

$$
\begin{aligned}
N_{\text {operations }}^{(\text {coaxial })} & =C N^{3} \sum_{m=-M}^{M}(M+1-|m|)^{3}=C N^{3}\left[(M+1)^{3}+2 \sum_{m=1}^{M} m^{3}\right] \\
& =C N^{3}\left[(M+1)^{3}+\frac{1}{2} M^{2}(M+1)^{2}\right]=\frac{1}{2} C N^{3}\left[(M+1)^{4}+(M+1)^{2}\right] .
\end{aligned}
$$

Therefore, for $M \gg 1$, we have

$$
\frac{N_{\text {operations }}^{(\text {general })}}{N_{\text {operations }}^{(\text {coaxial })}} \sim 2 M^{2} .
$$

which shows much higher efficiency of the algorithm for coaxial spheres.

Note also that in the case of coaxial spheres the number of the multipole reexpansion coefficients that need to be computed for each pair $p \neq q$ requires $O\left(M^{3}\right)$ operations, while in the general case such computations can be performed in $O\left(M^{4}\right)$ operations. These numbers are smaller than the leading order term in the complexity, that required for solution of linear equations, and thus Eqs. (61)-(63) provide a comparison between the two methods.

The above expressions for coaxial spheres assume that the $z$ axis coincides with the direction from the center of one of the spheres to the center of some other sphere. If coordinates of spheres are specified in the original reference frame, with the axis $z$ oriented arbitrarily with respect to the line connected the sphere centers we can rotate them so that the new reference frame is convenient for use with the coaxial algorithm.

\section{B. Computation of coaxial reexpansion coefficients}

Due to the symmetry relations (see Eqs. (49) and (51))

$$
(S \mid R)_{l n}^{m}=(S \mid R)_{l n}^{-m}, \quad m=0,1,2, \ldots
$$

the coaxial coefficients $(S \mid R)_{l n}^{m}\left(\mathbf{r}_{p q}^{\prime}\right)$ can be computed only for $l \geqslant n \geqslant m \geqslant 0$. The process of filling the matrix $\left\{(S \mid R)_{l n}^{m}\right\}$ can be performed efficiently using recurrence relations that first fill the layers with respect to the orders $l$ and $n$ followed by advancement with respect to the degree $m$. If such a procedure is selected then the first step is filling of the layer $m=0$. The initial value depends on the orientation of $\mathbf{r}_{p q}^{\prime}$ vector relative to the axis $\mathbf{i}_{z}$ (or $\mathbf{i}_{\widehat{z}}$ if rotation is performed), and is given by

$$
(S \mid R)_{l 0}^{0}\left(r_{p q}^{\prime}\right)=(S \mid R)_{l 0}^{00}\left(\mathbf{r}_{p q}^{\prime}\right)=\sqrt{(4 \pi)}(-1)^{l} S_{l}^{0}\left(\mathbf{r}_{p q}^{\prime}\right)=\epsilon_{l p q} \sqrt{(2 l+1)} h_{l}\left(k r_{p q}^{\prime}\right)
$$


where

$$
\epsilon_{l p q}=\left(-\frac{\mathbf{r}_{p q}^{\prime} \cdot \mathbf{i}_{z}}{\left|\mathbf{r}_{p q}^{\prime}\right|}\right)^{l}=\left\{\begin{array}{cr}
(-1)^{l}, & \text { for } \mathbf{r}_{p q}^{\prime} \cdot \mathbf{i}_{z}=\left|\mathbf{r}_{p q}^{\prime}\right| \\
1, & \text { for } \mathbf{r}_{p q}^{\prime} \cdot \mathbf{i}_{z}=-\left|\mathbf{r}_{p q}^{\prime}\right|
\end{array}\right.
$$

To advance with respect to the order $n$ at fixed $m \geqslant 0$ we can use Eq. (46) for $s=m$,

$$
a_{n}^{m}(S \mid R)_{l, n+1}^{m}=a_{n-1}^{m}(S \mid R)_{l, n-1}^{m}-a_{l}^{m}(S \mid R)_{l+1, n}^{m}+a_{l-1}^{m}(S \mid R)_{l-1, n}^{m}, \quad n=m, m+1, \ldots
$$

with the $a$ 's given by Eq. (47). For advancement with respect to $m$ it is convenient to use Eq. (41) for $s=m+1$,

$$
b_{m+1}^{-m-1}(S \mid R)_{l, m+1}^{m+1}=b_{l}^{-m-1}(S \mid R)_{l-1, m}^{m}-b_{l+1}^{m}(S \mid R)_{l+1, m}^{m}, \quad l=m+1, m+2, \ldots,
$$

with the $b$ 's given by Eq. (43) and then obtain the other $(S \mid R)_{l n}^{m+1}$ using Eq. (67).

\section{COMPUTATIONAL RESULTS}

We apply our method to several example problems. First, we compare the present technique with the BEM for problems involving two and three spheres, and show that the present method is accurate, and much faster. Next, we consider problems that, due to their size, would be impractical to handle with the BEM, and demonstrate that the present technique can deal with them. Convergence for these cases is demonstrated as the truncation number $M$ is increased.

\section{A. Single sphere}

For the case of a single sphere there are no multiple scattering effects. Despite this, the case is interesting from a practical viewpoint. We were unable to find this solution in the standard handbooks, and we include this limiting case to provide an analytical solution for both an arbitrary incident field and for a monopole source. From Eqs. (37) - (40) we have

$$
\begin{aligned}
\mathbf{L}^{(1)}= & \mathbf{I}, \mathbf{A}^{(1)}=\mathbf{D}^{(1)} \text {, or } \\
A_{n}^{(1) m} & =-\frac{k j_{n}^{\prime}(k a)+i \sigma j_{n}(k a)}{k h_{n}^{\prime}(k a)+i \sigma h_{n}(k a)} C_{n}^{(i n) m}\left(\mathbf{r}_{1}^{\prime}\right) .
\end{aligned}
$$

Here we will drop subscript 1 for $\sigma$ and $a$, while keeping them for the coordinates. Substituting this expression into Eq. (28) and Eq. (29), we obtain expressions for the potential 
and its normal derivative on the sphere surface as

$$
\begin{aligned}
\left.\psi\right|_{S_{1}} & =-\frac{1}{i k a^{2}} \sum_{l=0}^{\infty} \sum_{s=-l}^{l} \frac{C_{l}^{(i n) s}\left(\mathbf{r}_{1}^{\prime}\right) Y_{l}^{s}\left(\theta_{1}, \varphi_{1}\right)}{k h_{l}^{\prime}(k a)+i \sigma h_{l}(k a)} \\
\left.\frac{\partial \psi}{\partial n}\right|_{S_{1}} & =\frac{\sigma}{k a^{2}} \sum_{l=0}^{\infty} \sum_{s=-l}^{l} \frac{C_{l}^{(i n) s}\left(\mathbf{r}_{1}^{\prime}\right) Y_{l}^{s}\left(\theta_{1}, \varphi_{1}\right)}{k h_{l}^{\prime}(k a)+i \sigma h_{l}(k a)}=-\left.i \sigma \psi\right|_{S_{1}} .
\end{aligned}
$$

The coefficients $C_{n}^{(i n) m}\left(\mathbf{r}_{1}^{\prime}\right)$ are determined using Eq. (16) and depend on the incident field. In the case of a monopole source located at some point $\mathbf{r}=\mathbf{r}_{\text {source }}$, the incident field corresponds to the fundamental solution of the Helmholtz equation

$$
\psi_{\text {in }}(\mathbf{r})=Q G_{k}\left(\mathbf{r}-\mathbf{r}_{\text {source }}\right)=Q \frac{e^{i k\left|\mathbf{r}-\mathbf{r}_{\text {source }}\right|}}{4 \pi\left|\mathbf{r}-\mathbf{r}_{\text {source }}\right|},
$$

where $Q$ is the source intensity (complex, if the phase $\Phi$ is not zero)

$$
Q=|Q| e^{i \Phi}
$$

For multiple spheres, expansion of this function near the center of the $q$ th sphere $\mathbf{r}=\mathbf{r}_{q}^{\prime}$ (see Fig. 2) can be found elsewhere (e.g., Morse and Feshbach, 1953)

$$
\psi_{\text {in }}(\mathbf{r})=Q i k \sum_{n=0}^{\infty} \sum_{m=-n}^{n} S_{n}^{-m}\left(\mathbf{r}_{\text {source }}-\mathbf{r}_{q}^{\prime}\right) R_{n}^{m}\left(\mathbf{r}_{q}\right), \quad\left|\mathbf{r}_{q}\right| \leqslant\left|\mathbf{r}_{\text {source }}-\mathbf{r}_{q}^{\prime}\right| .
$$

Comparing Eqs. (16) and (74) we obtain

$$
C_{n}^{(i n) m}\left(\mathbf{r}_{q}^{\prime}\right)=Q i k S_{n}^{-m}\left(\mathbf{r}_{\text {source }}-\mathbf{r}_{q}^{\prime}\right)
$$

A simplification of the general formula for $C_{n}^{(i n) m}$, Eq. (75), is possible, since the problem for monopole source and a single sphere is axisymmetric relative to the axis connecting the sphere center and the source location. Setting the axis $z_{1}$ to be this axis, we have

$$
C_{n}^{(i n) m}=Q i k \delta_{m 0} h_{n}\left(k\left|\mathbf{r}_{\text {source }}-\mathbf{r}_{1}^{\prime}\right|\right) Y_{n}^{0}(0,0)=Q i k \delta_{m 0} \sqrt{\frac{2 n+1}{4 \pi}} h_{n}(k d),
$$

where $d$ is the distance between the source and the sphere center. The expressions for the surface values of the potential and its derivative Eqs. (70) and (71) become

$$
\begin{aligned}
\left.\psi\right|_{S_{1}} & =-\frac{Q}{4 \pi a^{2}} \sum_{l=0}^{\infty} \frac{(2 l+1) h_{l}(k d) P_{l}\left(\cos \theta_{1}\right)}{k h_{l}^{\prime}(k a)+i \sigma h_{l}(k a)} \\
\left.\frac{\partial \psi}{\partial n}\right|_{S_{1}} & =\frac{i Q \sigma_{1}}{4 \pi a^{2}} \sum_{l=0}^{\infty} \frac{(2 l+1) h_{l}(k d) P_{l}\left(\cos \theta_{1}\right)}{k h_{l}^{\prime}(k a)+i \sigma h_{l}(k a)}=-\left.i \sigma \psi\right|_{S_{1}} .
\end{aligned}
$$


For the particular case of a sound-hard $\left(\sigma_{1}=0\right)$ surfaces this gives

$$
\left.\psi\right|_{S_{1}}=-\frac{Q}{4 \pi k a^{2}} \sum_{l=0}^{\infty}(2 l+1) \frac{h_{l}(k d)}{h_{l}^{\prime}(k a)} P_{l}\left(\cos \theta_{1}\right),\left.\quad \frac{\partial \psi}{\partial n}\right|_{S_{q}}=0
$$

while for sound-soft $\left(\sigma_{1}=\infty\right)$ surfaces we have

$$
\left.\psi\right|_{S_{1}}=0,\left.\quad \frac{\partial \psi}{\partial n}\right|_{S_{1}}=\frac{Q}{4 \pi a^{2}} \sum_{l=0}^{\infty}(2 l+1) \frac{h_{l}(k d)}{h_{l}(k a)} P_{l}\left(\cos \theta_{1}\right) .
$$

These limiting cases are classical and their expressions can be found elsewhere (e.g. see Hanish (1981)).

We compared results of computations of the potential on the surface with those provided by Eq. (79) and that obtained using the BEM, as realized in the software package COMET. The figures below illustrate comparisons of the following transfer function $H$ measured in $\mathrm{dB}$, which represents the ratio of the amplitude of the acoustic field at specified location of the surface to the amplitude of the incident field at the center of the sphere:

$$
H=20 \lg \left|\frac{\left.\psi\right|_{S_{1}}}{\psi_{\text {in }}\left(\mathbf{r}_{1}^{\prime}\right)}\right|
$$

The solution, Eq. (79), for a single sphere was used by Duda and Martens (1998), for an investigation of scattering cues in audition. Figure 3 shows good agreement between computations using all methods. In this example we include also computations for different impedances of the sphere.

We also tested the results of computations obtained using different truncation numbers. Duda and Martens (1998) used truncation based on comparison of subsequent terms in the series (in this particular case the series was truncated when the ratio of such terms is smaller than $10^{-6}$ ). Experiments showed that excellent agreement with these results is achieved, if the truncation number is selected using the heuristic

$$
M=[e k a], \quad e=2.71828 \ldots
$$

which shows that it increases with the wavenumber. For large $k a$ good agreement was observed for $M \geqslant\left[\frac{1}{2} e k a\right]$, while differences were visible for smaller $M$. For moderate $k a$ such differences appeared for truncation numbers below the value provided in Eq. (82). 


\section{B. Two spheres}

Since there is no closed-form analytical solution for two spheres in a simple form, we compared the numerical results obtained using our code, and by using the BEM. As an example we considered computation of the function Eq. (81).

The model consists of two spheres, which are touching at one point. In the computations the ratio of sphere radii was taken to be 1.3253. The origin of the reference frame was located at the center of the smaller sphere and the direction of the $y$-axis was from the larger sphere to the smaller one. The $z$-axis was directed towards a monopole source, generating the incident field, which was located at the distance of 10 radii from the smaller sphere. The frequency of the incident wave nondimensionalized with the radius of the smaller sphere corresponded to $k a_{1}=3.0255$. The mesh for computations using the BEM contained 5400 triangular elements (2700 elements for each sphere). A picture of this two sphere configuration with computational mesh and distribution of the acoustic pressure is shown in Fig. 4. In the computations the impedances of both spheres were set to zero.

For computations using MultisphereHelmholtz the truncation number was automatically set to

$$
M=\left[\frac{1}{2} e k r_{12}^{\prime}\right]
$$

For the above given values of $a_{2} / a_{1}$ and $k a_{1}$ this provided $M=9$, so that the total number of modes $n$ in the multipole expansion was 10 for each sphere (or $100 A_{n}^{m}$ coefficients for each sphere).

Figure 5 shows a comparison between the BEM and the MultisphereHelmholtz computational results for function $H$ calculated for sphere 1 according to Eq. (81). Each curve corresponds to a fixed value of the spherical polar angle $\theta_{1}$ and demonstrates dependence on the angle $\varphi_{1}$. Note that dependence on the angle $\varphi_{1}$ is only due to the presence of the sphere 2. The comparison shows a good agreement between the results obtained by different methods. Some small dispersion of the points obtained using BEM is due to the mesh discretization of the sphere surface, which normally can be avoided by additional smoothing/interpolation procedures (we did not apply such smoothing in the results plotted). Our code far outperformed BEM computationally, both in achieving much faster computations (seconds as opposed to tens of minutes on the same computer) and memory usage. 
Figure 7 demonstrates computations of $H$ for the two sphere geometry with the parameters described above, but for a higher wavenumber, and with different impedances for the larger sphere. For the given geometry, the automatically selected truncation number was $M=31$. This number is large enough to observe a substantial difference in speed of computations and memory usage between the general algorithm and the coaxial one (see Eq. $(63))$.

Proper selection of the truncation number is an important issue for applications of multipole translation techniques. Figure 6 shows convergence of the computations with increasing truncation numbers for $H$ at a specified point on the surface $\left(\theta_{1}=60^{\circ}\right.$ and $\varphi_{1}=0^{\circ}$ in the case shown in the figure). Computations with low truncation numbers may provide poor accuracy. At some particular truncation number (depending on the non-dimensional wavenumber $k a$ ) the computational results stabilize (note that since the solution $H$ depends on the wavenumber, and so for each $k a$ the solution asymptotes to the corresponding value). Further increase in the truncation number increases both the accuracy of the results and computational time/memory, since the matrix size grows in proportion to $M^{4}$ in the general case, and to $M^{3}$ for coaxial spheres. However, at some truncation numbers, which slightly exceed the value provided by Eq. (83), the computations can encounter difficulties that arise from the exponential growth of portions of the terms in the expansions, leading to overflow related errors. These are due to the spherical Hankel functions of large order, $h_{n}\left(k r_{p q}^{\prime}\right)$, entering the reexpansion system matrix. Asymptotic expansion of the Hankel function at large $n$ and fixed $k r_{12}^{\prime}$ shows, that the growth starts at $n \sim \frac{1}{2} e k r_{12}^{\prime}$. This is used as the basis for automatic selection of the truncation number Eq. (83). Of course, this limitation is purely based on the order of computation of the elements, and the product of terms remain finite, and calculations can be performed for much larger $M$ than given by Eq. (83). However, we have not yet modified our software to use this order of computation.

The computations presented in Fig. 6 show that the actual stabilization occurs at smaller $M$ than those given by Eq. (83) (where we have for $k a_{1}=1,5,10,20,30$ the following values: $M=3,15,31,63,94$, respectively. Results of our numerical experiments show that for large $k r_{12}^{\prime}$ reasonable accuracy can be achieved at $M^{*} \sim \frac{1}{2} M_{\max }$, where $M_{\max }$ is provided by Eq. (83). At the same time, for lower $k r_{12}^{\prime}$ formula (83) provides values which cannot be reduced, and accurate computations can be achieved with $M$ slightly larger than $M_{\min }$, with $M_{\min }$ is provided by Eq. (83). 
In a recent paper (Gumerov and Duraiswami, 2001b) we presented results of the computation of the surface potential for a sphere near a rigid wall. In this case the rigid wall could be replaced by an image sphere and an image source and the coaxial multipole reexpansion can be used. The problem of sound scattering by a sphere near a rigid wall is in some sense a simplification of the general problem for two spheres, since both the real and the image spheres in this case have the same radius and impedance. For this case, the coefficients of the multipole expansions near each sphere $A_{n}^{(1) m}$ and $A_{n}^{(2) m}$ are symmetrical and the dimension of the system can be reduced using this symmetry by a factor of two. In that paper, we also provided a study and discussion of the influence of the distance between the sphere and the wall and frequency of the field on the surface potential.

\section{Three arbitrarily located spheres}

If the cases of one and two spheres can be covered using simplified codes, the case of three non-coaxial spheres requires the general three-dimensional multipole translation. As in the case of two spheres discussed above, we compared results of computations for three spheres using MultisphereHelmholtz and the COMET BEM software.

For this computational example we placed an additional sphere (3) to the case described above. The distance between the centers of spheres 1 and 3 was the same as the distance between the centers of spheres 1 and 2. The parameters of the incident field were the same as for the case of two spheres. The mesh for computations using the BEM contained 5184 triangular elements, 1728 elements for each sphere. A picture of this configuration with the computational mesh and distribution of the acoustic pressure is shown in Fig. 8. In the computations the three spheres were all taken to be sound-hard.

Results of comparisons between BEM and MultisphereHelmholtz computations with $M=$ 9 are shown in Fig. 9. The comparison is as good as in the case of two spheres. Since Figs. 5 and 9 represent similar dependences, we can notice that the presence of the third sphere reduced (at some points by $3-4 \mathrm{~dB}$ ) the amplitude of the sound field on sphere 1 . 


\section{Many spheres}

The case of sound and electromagnetic wave scattering by many arbitrarily located spheres has numerous practical and theoretical applications, including acoustics and hydrodynamics of multiphase flows, sound propagation in composite materials, electromagnetic waves in clouds, and inverse problems, such as the detection of buried objects, medical tomography, etc.

We considered the following hypothetical problem of sound scattering by a screen of spherical particles. The geometry of the problem is shown in Fig. 10. Here the incident field is generated by a monopole source located at distance $d$ from a flat particle screen of thickness $\delta$. A sensor (e.g., a microphone) measuring the acoustic pressure is located behind the screen at the same distance as the source. $N$ spheres with the same acoustic impedance (in the examples below we took the spheres as sound-hard), but with possibly different sizes are distributed according to some distribution density over their radii, and, have locations of their centers within a box $\delta \times h \times h$ representing the screen. The objective is to evaluate the effect of the screen on the sound propagation.

This effect can be evaluated by defining a "Screen Transfer Function" (STF), which we define as

$$
S T F=20 \lg \left|\frac{\psi\left(\mathbf{r}_{m i c}^{\prime}\right)}{\psi_{i n}\left(\mathbf{r}_{m i c}^{\prime}\right)}\right|,
$$

where $\mathbf{r}_{m i c}^{\prime}$ is the radius-vector of the microphone location and which is measured in decibels.

Two examples of computations with many spheres are presented below. In the first example we placed 16 spheres with uniform random distribution of their dimensionless radii from $a_{\min }=0.5$ to $a_{\max }=1.5$. Dimensionless parameters of the screen were $d=10, \delta=5$, and $h=50$. The sphere centers were distributed uniformly within the screen. The view of this screen in the $y z$-plane is shown in Fig. 11. In the computations we chose three different wavenumbers, $k a=1, k a=3$, and $k a=5$, where $a$ is the length scale (the characteristic sphere radius). Computations were performed using the general software with increasing truncation number $M$. Dependences of the STF on $M$ at various $k a$ are shown in Fig. 12 . It is seen that results converge to some value depending on $k a$. This type of check shows that for the given geometry relatively low truncation numbers can be used, which are much smaller than given by Eq. (83), where instead of $r_{12}^{\prime}$ some representative intersphere distance 
$r_{p q}^{\prime}$ is selected. We noticed however, that in some cases when we have many spheres with very different intersphere distances (from touching spheres, to spheres located at large $k r_{p q}^{\prime}$ ), stabilization of computation only occurs at higher truncation numbers than prescribed by Eq. (83). Thus, at this point the procedure we recommend is an experimental one to check the results of several calculations and find the truncation number at which results converge.

In the second example we put a regular monolayer screen $(d=10, \delta=0$, and $h=50)$ of 121 spheres of the same radii $a=1$, which centers form a regular grid in plane $x=d$ (see Fig. 13). Again we compared results obtained with the aid of MultisphereHelmholtz at increasing $M$ and different wavenumbers and found fast convergence (see Fig. 14).

\section{CONCLUSIONS}

We have developed a procedure for solution of the Helmholtz equation for the case of $N$ spheres of various radii and impedances arbitrary located in three dimensional space. This solution uses a T-matrix method where the matrices are computed using a multipole reexpansion technique. We presented computational results for two and three spheres obtained both using the Boundary Element Method with fine discretization of the surfaces (thousands of elements), and showed that our solutions are correct. For the case of larger numbers of spheres, we demonstrated that our results are consistent, by showing that they converge as the truncation number increases.

An open problem that remains is the proper choice of the truncation number as a function of the wavenumber, sphere sizes, and intersphere distances. In case the truncation number is properly selected, the solution showed high accuracy, and substantial speed-up in comparison to the Boundary Element Method. In cases when the centers of the spheres are located on a line (which is always true for two spheres, and can be realized in particular problems for $N$ spheres) computations using a coaxial multipole reexpansion can be performed with much higher efficiency, than in the general case.

Future improvements to the method proposed here may include use of spatial grouping of nearby spheres into clusters represented by one multipole expansion, variable truncation numbers depending on the particular distribution of spheres, implementation of a fast multipole method based evaluation of the matrix-vector product in Eq. (39), and development of iterative algorithms for solution of that equation. 


\section{ACKNOWLEDGMENTS}

We would like to gratefully acknowledge the support of NSF award 0086075. We would also like to thank Dr. S.T. Raveendra of Collins and Aikman Automotive Interior Systems, Plymouth, MI for providing us with the COMET BEM software. We also thank the reviewers of this paper for pointing out missing references, prior work, and for their helpful comments.

\section{References}

Algazi, V.R., Duda, R.O., Duraiswami, R., Gumerov, N.A., and Tang, Z., (2002), “Approximating the head-related transfer function using simple geometric models of the head and torso," accepted, J. Acoust. Soc. Am.

Brunning, J.H., and Lo, Y.T., (1971), "Multiple scattering of EM waves by spheres, parts I and II," IEEE Trans. Antennas Propagat., AP-19, 3, 378-400.

Chew, W.C., (1992), "Recurrence relations for three-dimensional scalar addition theorem," J. Electromagnetic Waves and Applications, 6(2), 133-142.

Duda, R.O., and Martens, W.L., (1998), "Range dependence of the response of a spherical head model," J. Acoust. Soc. Am. 104, 3048-3058.

Duraiswami, R., and Prosperetti, R., (1995), "Linear Pressure Waves in Fogs," J. Fluid Mech., 299, pp. 187-215.

Duraiswami, R., Gumerov, N.A., Davis, L., Shamma, S.A., Elman, H.C., Duda, R.O., Algazi, V. R., Liu, Q-H., and. Raveendra, S. T., (2000), "Individualized HRTFs using computer vision and computational acoustics," J. Acoust. Soc. Am., 108, 2597. Also available on the web at http://www.acoustics.org/press/140th/duraiswami.htm

Epton, M.A., and Dembart, B., (1995), "Multipole translation theory for the threedimensional Laplace and Helmholtz equations," SIAM J. Scientific Computing, 4, 865-897.

Gaunaurd, G.C. and Huang, H., (1994), "Acoustic scattering by a spherical body near a plane boundary," J. Acoust. Soc. Am., 96(4), 2525-2536.

Gaunaurd, G.C., Huang, H., and Strifors, H., (1995), "Acoustic scattering by a pair of spheres," J. Acoust. Soc. Am., 98(1), 495-507.

Gumerov, N.A., Ivandaev, A.I., and Nigmatulin, R.I., (1988), "Sound waves in monodisperse gas-particle or vapour-droplet mixtures." J. Fluid Mech., 193, 53-74. 
Gumerov, N.A., and Duraiswami, R., (2001), "Fast, Exact, and Stable Computation of Multipole Translation and Rotation Coefficients for the 3-D Helmholtz Equation," University of Maryland Institute for Advanced Computer Studies Technical Report UMIACS-TR-\# 2001-44, Also CS-TR-\# 4264. (Available at http://www.cs.umd.edu/Library/TRs/CS-TR4264/CS-TR-4264.pdf)

Gumerov, N.A., and Duraiswami, R., (2001b), "Modeling the effect of a nearby boundary on the HRTF," Proc. International Conference on Acoustics, Speech, and Signal Processing 2001, Salt Lake City, Utah, May 7-11, 2001, 5, 3337-3340.

Gumerov, N.A., Duraiswami, R., Tang, Z., (2002), "Numerical study of the influence of the torso on the HRTF," to appear in Proc. of the International Conference on Acoustics, Speech and Signal Processing, Orlando, Florida, 2002.

Hanish, S., (1981), A Treatise on Acoustical Radiation, Naval Research Laboratory, Washington, DC, pp. 123-125.

Huang, H. and Gaunaurd, G.C., (1995), "Acoustic scattering of a plane wave by two spherical elastic shells," J. Acoust. Soc. Am., 98(4), 2149-2156.

Kahana,Y., (2001), "Numerical modelling of the head-related transfer function," Ph.D. thesis, University of Southampton.

Katz, B.F.G., (2001), "Boundary element method calculation of individual head-related transfer function. I. Rigid model calculation," J. Acoust. Soc. Am., 110, 2440-2448.

Koc, S., Song, J., and Chew, W.C., (1999), "Error analysis for the numerical evaluation of the diagonal forms of the scalar spherical addition theorem," SIAM J. Numer. Anal., 36, 906-921.

Koc, S., and Chew, W.C., (1998), "Calculation of acoustical scattering from a cluster of scatterers," J. Acoust. Soc. Am., 103, 721-734.

Marnevskaya, L.A., (1969), "Diffraction of a plane scalar wave by two spheres," Soviet Physics-Acoustics, 14(3), 356-360.

Marnevskaya, L.A. (1970), "Plane wave scattering by two acoustically-rigid spheres," Soviet Physics-Acoustics, 15(4), 499-502.

Mishchenko, M. I., Travis, L.D., and Mackowski, D.W., (1996), "T-matrix computations of light scattering by nonspherical particles: a review," J. Quant. Spectrosc. Radiat. Transfer, 55, 535-575.

Morse, P.M., and Feshbach, H., (1953), Methods of Theoretical Physics -I, McGraw-Hill. 
Peterson, B., and Strom, A., (1974), " Matrix formulation of acoustic scattering from an arbitrary number of scatterers," J. Acoust. Soc. Am., 56, 771-780.

Rokhlin, V., (1993), "Diagonal forms of translation operators for the Helmholtz equation in three dimensions," Appl. and Comp. Harmonic Analysis, 1, 82-93.

Varadan, V.K., and Varadan, V.V., (1980), Acoustic, Electromagnetic and Elastic Wave Scattering: Focus on the T-Matrix Approach., New York: Pergamon Press.

Waterman, P. C., and Truell, R., (1961), "Multiple scattering of waves," J. Math. Phys., $\mathbf{2}, 512-537$. 


\section{List of Figures}

1 The multiple scattering problem considered in this paper. ptmu.ptmu

2 Notation denoting the different reference frames used in the multipole reexpansion technique. ptmu. ptmu ptmu. ptmu ptmu. ptmu ptmu.ptmu

3 The normalized surface transfer function $H$, Eq. (81), for a single sphere of radius $a=8.25 \mathrm{~cm}$ and a monopole source, located at a distance $d / a=10$ from the center of the sphere for spheres of different impedances $\sigma / k$ (shown near curves). The continuous and dashed lines show results of computations using the present method with truncation number $M=8$. Circles and diamonds respectively show the results of Duda \& Martens (1998) and of BEM computations for $\sigma=0$. In the BEM the sphere surface was discretized using 2700 linear elements. $\quad$ ptmu. ptmu ptmu. ptmu ptmu. ptmu ptmu. ptmu

4 An example of BEM (COMET 4.0) computations of potential distribution over the surface of two spheres generated by a monopole source. Each sphere surface is discretized to 2700 triangular elements. The ratio of sphere radii is 1.3253 and they touch in one point. ptmu. ptmu ptmu. ptmu ptmu. ptmu

5 Comparison of the angular dependence of function $H$, Eq. (81), over the surface of a smaller sphere computed for the two sphere geometry shown in Fig. 4 using the BEM and the multipole reexpansion technique. Both spheres have zero impedance. ptmu. ptmu ptmu. ptmu ptmu. ptmu ptmu.ptmu

6 Dependence of the compuations of $H$, Eq. (81), for the two sphere geometry (see Fig. 4) on the truncation number $M$ for different non-dimensional wavenumbers. ptmu. ptmu ptmu. ptmu ptmu. ptmu ptmu. ptmu ptmu. ptmu

7 Angular dependence of $H$ in Eq. (81) over the surface of the sound-hard smaller sphere for the geometry shown in Fig. 4 for different impedances of the larger sphere. Results computed using the present method with automatically selected truncation number $M=31 . \quad$ ptmu. ptmu ptmu. ptmu ptmu. ptmu 
8 BEM (COMET 4.0) computations of the potential distribution over the surface of three spheres generated by a monopole source. Each sphere surface is discretized using 1728 triangular elements. Two spheres of non-dimensional radii 1 (sphere 1) and 1.3253 (sphere 2) touch at one point. Sphere 3, with non-dimensional radius 1 is located at a distance 2.3253 from the center of sphere 1 , on the line connecting the source and the center of sphere 1.

9 Comparison of the angular dependence of $H(81)$ over the surface of smaller sphere computed for the three sphere geometry shown in Fig. 8 using the BEM and the multipole reexpansion technique. All three spheres have sound-hard surfaces. $\quad$ ptmu. ptmu ptmu. ptmu ptmu. ptmu ptmu. ptmu ptmu. ptmu

10 A sketch of the problem geometry for sound propagation through a screen of spherical particles. ptmu. ptmu ptmu. ptmu ptmu. ptmu ptmu. ptmu

11 A scattering screen of 16 spheres with random sizes and random locations of their centers. The centers are uniformly distributed inside a box $10<x<15$, $-25<y<25,-25<z<25$. The radii are uniformly distributed over $0.5<a<1.5$. ptmu. ptmu ptmu. ptmu ptmu. ptmu ptmu. ptmu ptmu. ptmu

12 Convergence test for the problem of sound scattering by a screen of 16 randomly sized spheres with random location of their centers (as shown in Figure 11). Three different curves computed at different $k a$, where $a$ is the mean of the sphere radii distribution. ptmu. ptmu ptmu. ptmu ptmu. ptmu

13 The view of a scattering screen of 121 spheres of the same size $a=1$ and location of their centers at the nodes of a square grid $-25<y<25,-25<$ $z<25$ with the grid size $\Delta y=\Delta z=5$. ptmu. ptmu ptmu. ptmu ptmu. ptmu

14 Convergence test for the problem of sound scattering by a screen of 121 equal sized spheres located at the nodes of a regular grid (as shown in Fig. 13). Three different curves are computed at different $k a$ indicated near the curves. 


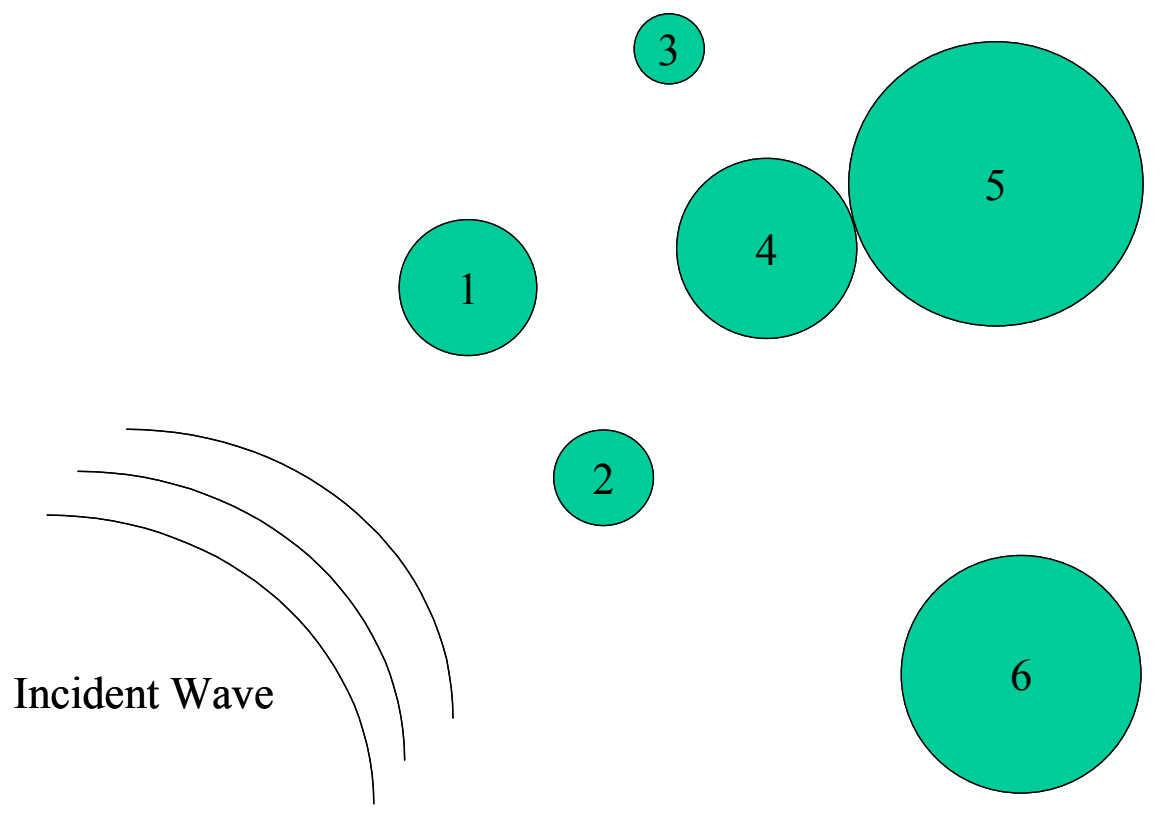

FIG. 1: The multiple scattering problem considered in this paper. 


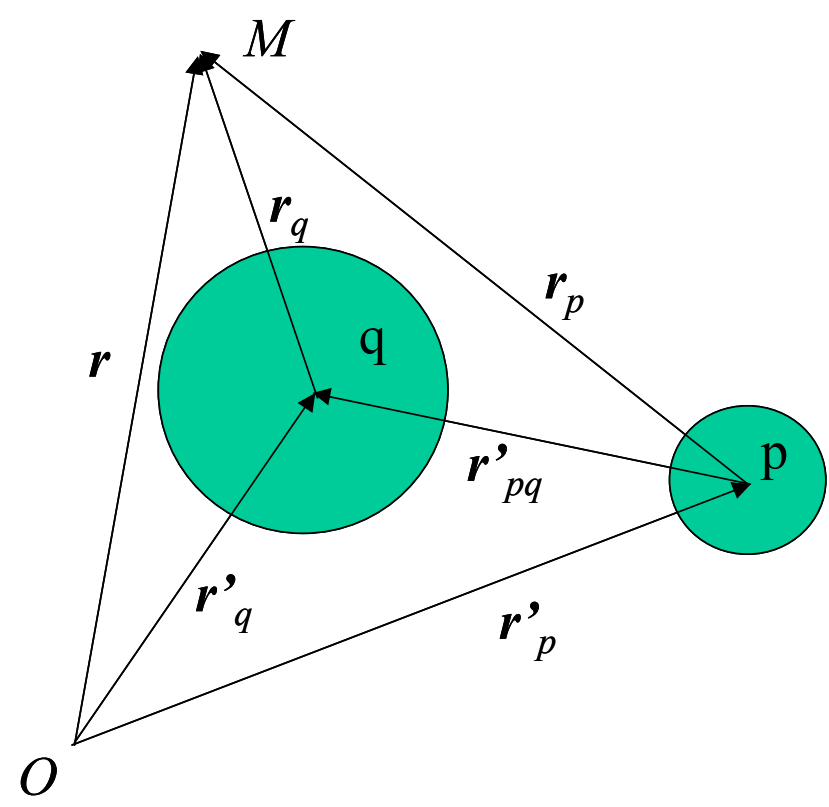

FIG. 2: Notation denoting the different reference frames used in the multipole re-expansion technique. 


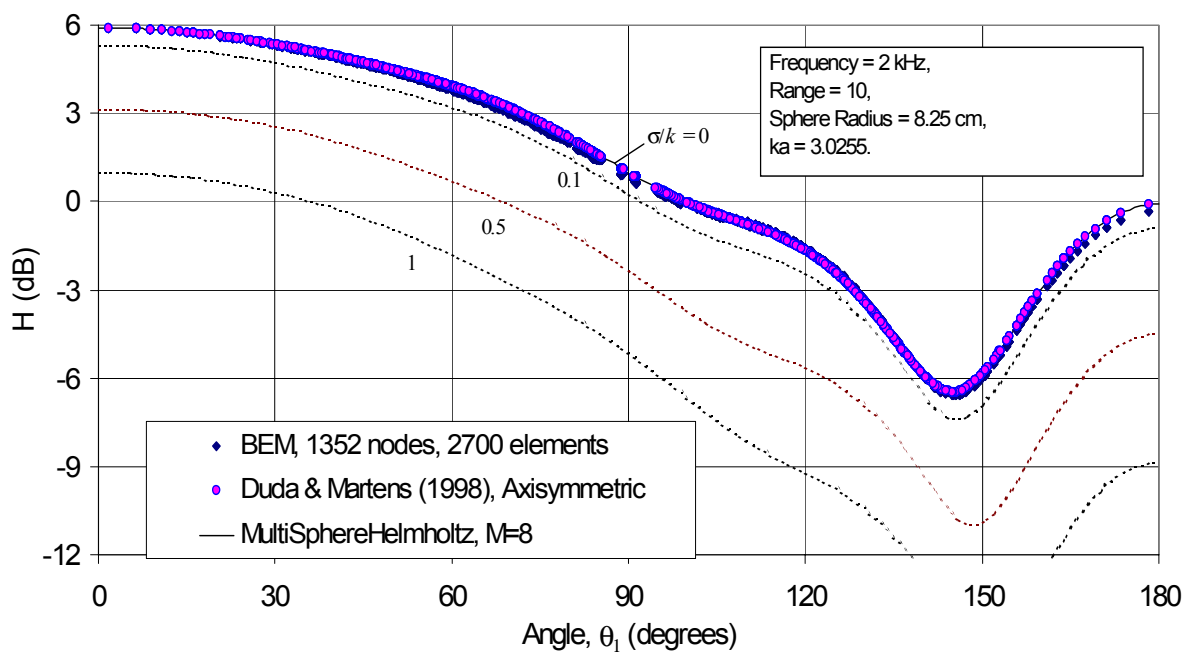

FIG. 3: The normalized surface transfer function $H$, Eq. (81), for a single sphere of radius $a=8.25$ $\mathrm{cm}$ and a monopole source, located at a distance $d / a=10$ from the center of the sphere for spheres of different impedances $\sigma / k$ (shown near curves). The continuous and dashed lines show results of computations using the present method with truncation number $M=8$. Circles and diamonds respectively show the results of Duda \& Martens (1998) and of BEM computations for $\sigma=0$. In the BEM the sphere surface was discretized using 2700 linear elements. 


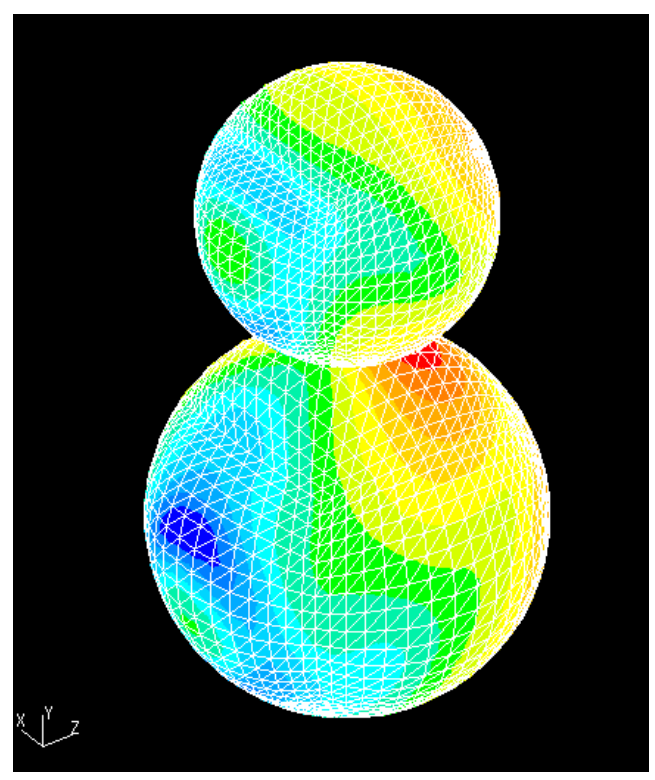

FIG. 4: An example of BEM (COMET 4.0) computations of potential distribution over the surface of two spheres generated by a monopole source. Each sphere surface is discretized to 2700 triangular elements. The ratio of sphere radii is 1.3253 and they touch in one point. 


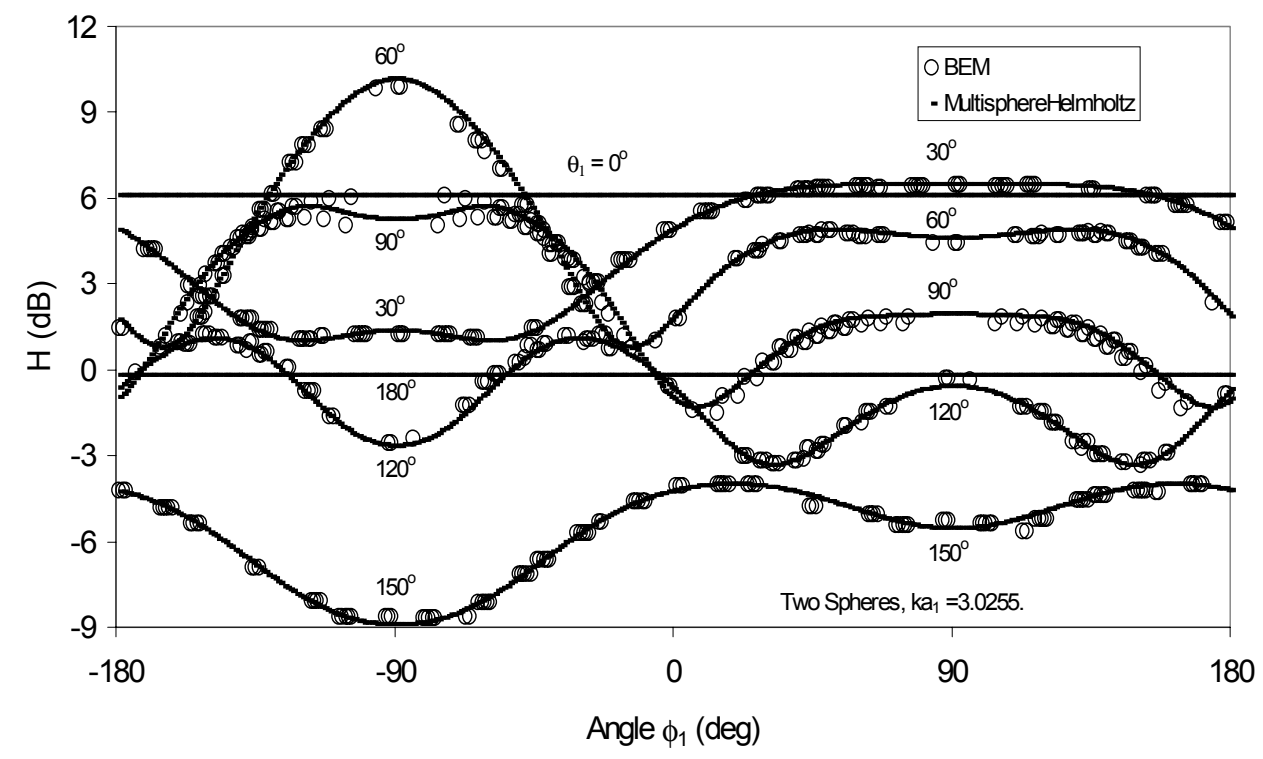

FIG. 5: Comparison of the angular dependence of function $H$, Eq. (81), over the surface of a smaller sphere computed for the two sphere geometry shown in Fig. 4 using the BEM and the multipole reexpansion technique. Both spheres have zero impedance. 


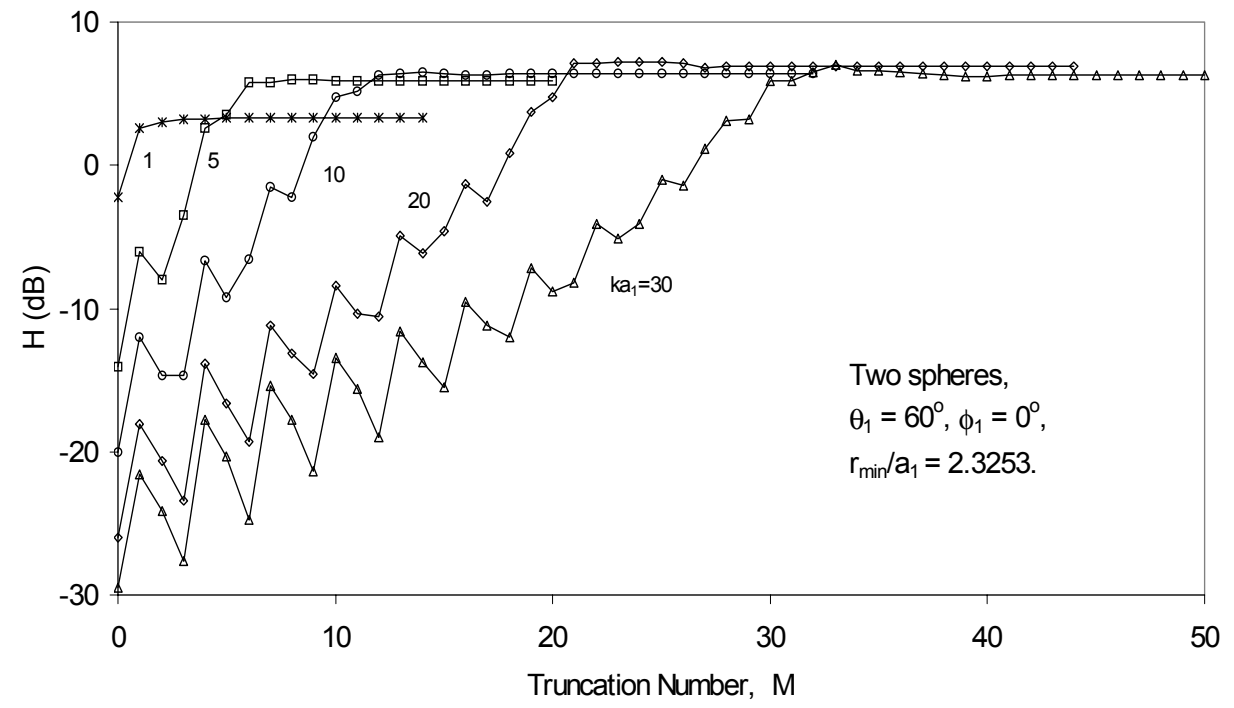

FIG. 6: Dependence of the compuations of $H$, Eq. (81), for the two sphere geometry (see Fig. 4) on the truncation number $M$ for different non-dimensional wavenumbers. 


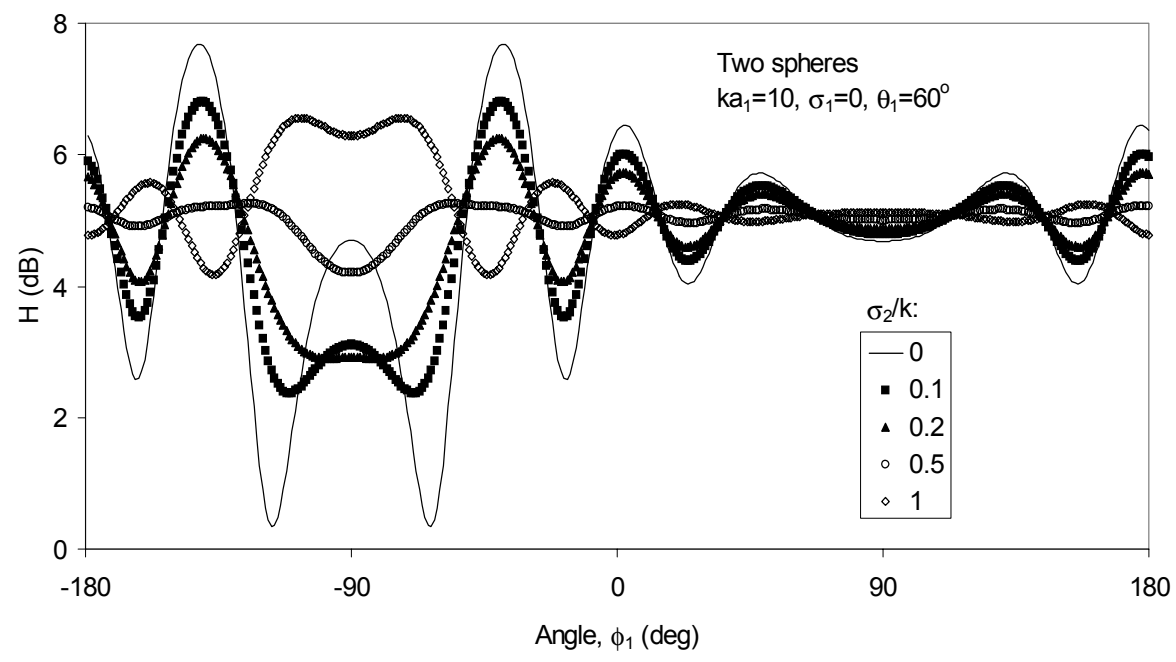

FIG. 7: Angular dependence of $H$ in Eq. (81) over the surface of the sound-hard smaller sphere for the geometry shown in Fig. 4 for different impedances of the larger sphere. Results computed using the present method with automatically selected truncation number $M=31$. 


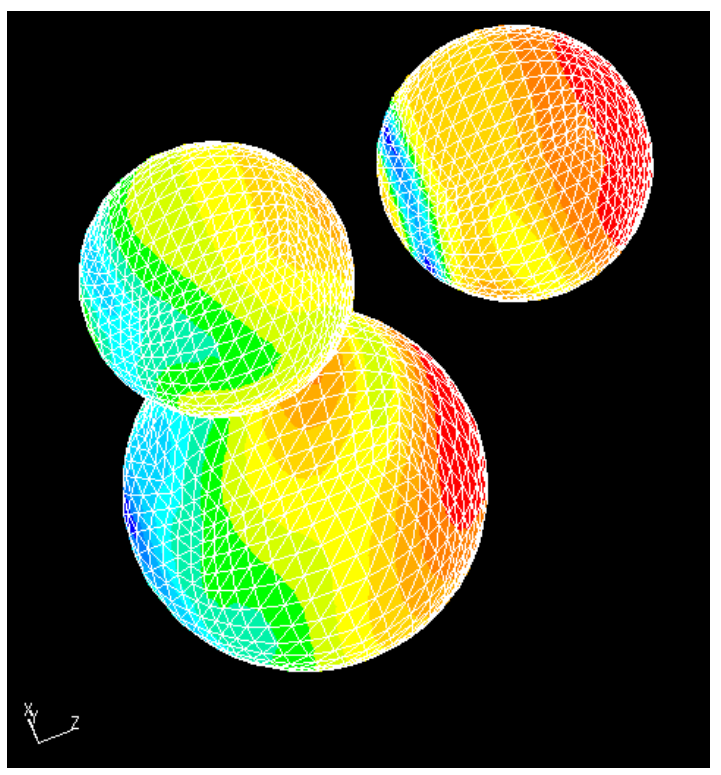

FIG. 8: BEM (COMET 4.0) computations of the potential distribution over the surface of three spheres generated by a monopole source. Each sphere surface is discretized using 1728 triangular elements. Two spheres of non-dimensional radii 1 (sphere 1) and 1.3253 (sphere 2) touch at one point. Sphere 3, with non-dimensional radius 1 is located at a distance 2.3253 from the center of sphere 1 , on the line connecting the source and the center of sphere 1. 


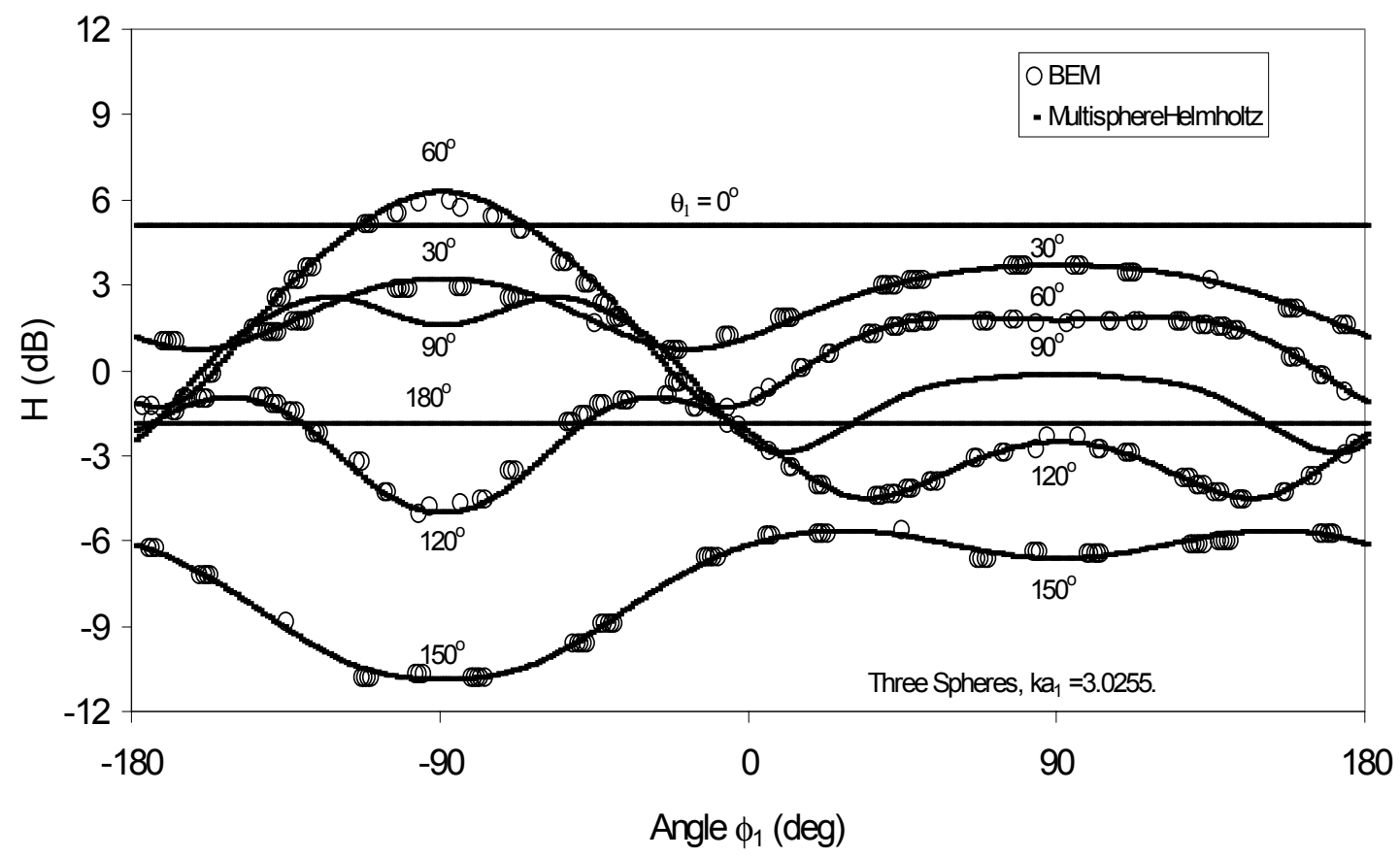

FIG. 9: Comparison of the angular dependence of $H$ (81) over the surface of smaller sphere computed for the three sphere geometry shown in Fig. 8 using the BEM and the multipole reexpansion technique. All three spheres have sound-hard surfaces. 


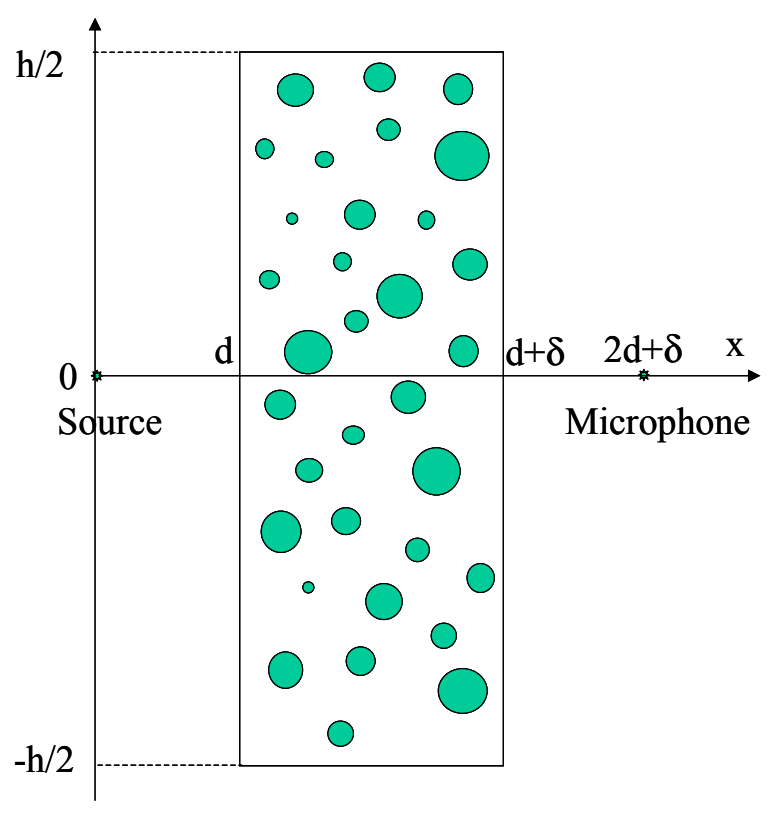

FIG. 10: A sketch of the problem geometry for sound propagation through a screen of spherical particles. 


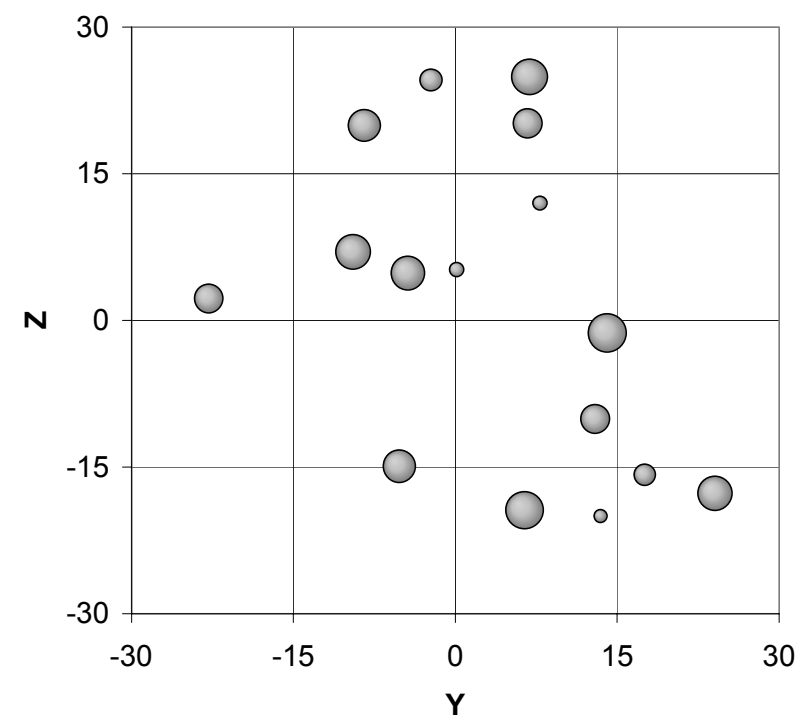

FIG. 11: A scattering screen of 16 spheres with random sizes and random locations of their centers. The centers are uniformly distributed inside a box $10<x<15,-25<y<25,-25<z<25$. The radii are uniformly distributed over $0.5<a<1.5$. 


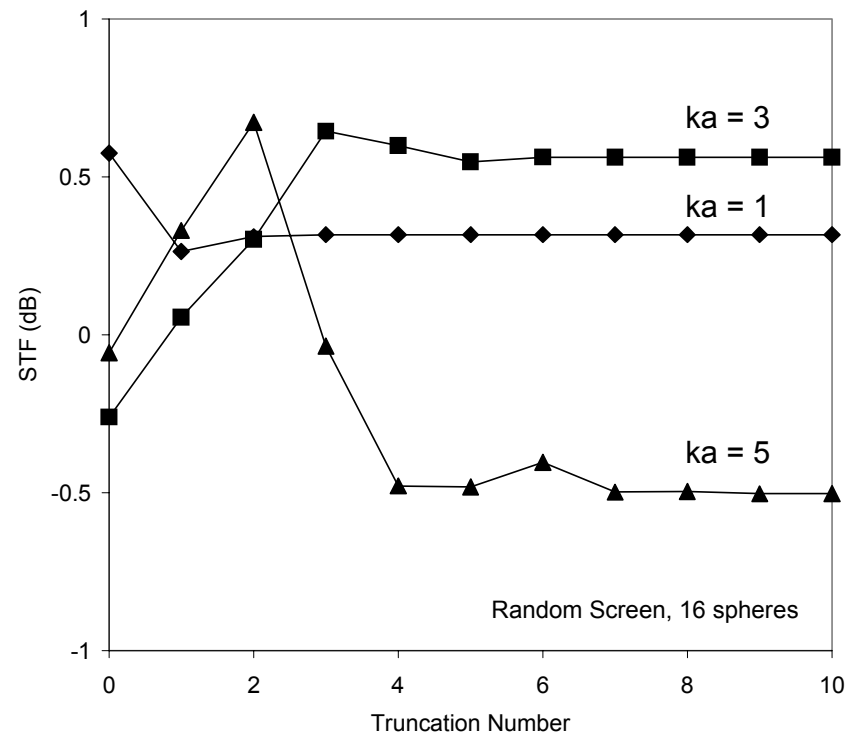

FIG. 12: Convergence test for the problem of sound scattering by a screen of 16 randomly sized spheres with random location of their centers (as shown in Figure 11). Three different curves computed at different $k a$, where $a$ is the mean of the sphere radii distribution. 


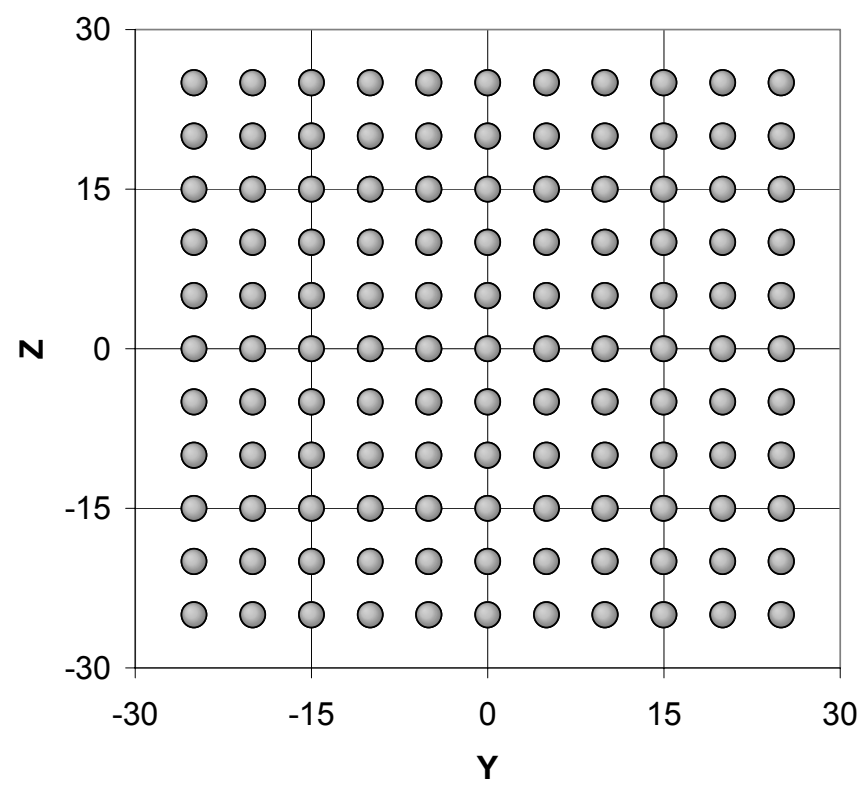

FIG. 13: The view of a scattering screen of 121 spheres of the same size $a=1$ and location of their centers at the nodes of a square grid $-25<y<25,-25<z<25$ with the grid size $\Delta y=\Delta z=5$. 


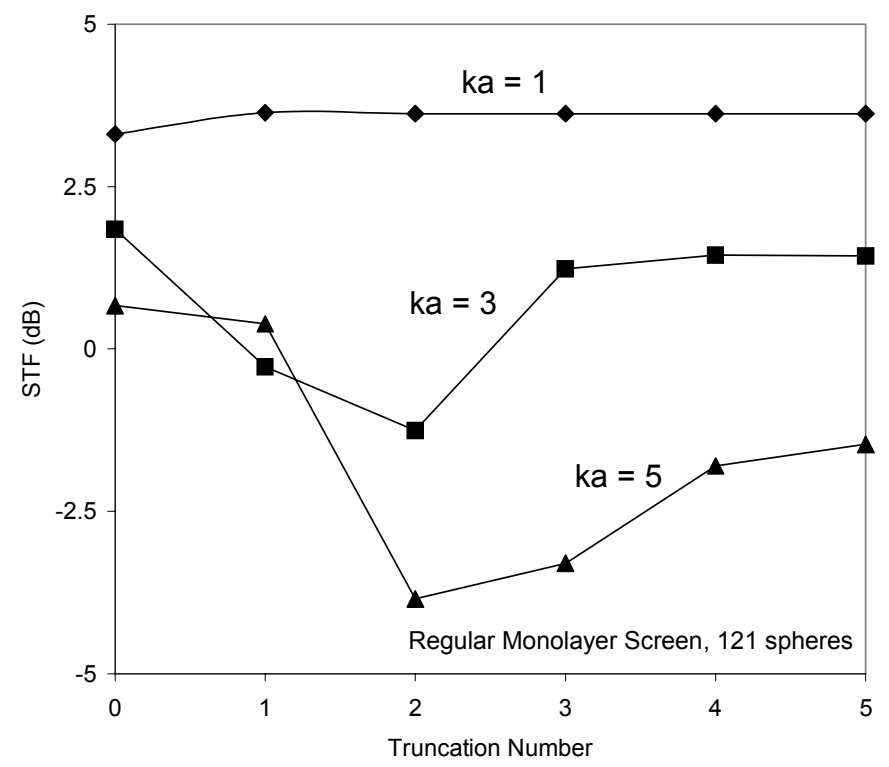

FIG. 14: Convergence test for the problem of sound scattering by a screen of 121 equal sized spheres located at the nodes of a regular grid (as shown in Fig. 13). Three different curves are computed at different $k a$ indicated near the curves. 